\title{
A Structural and Genotypic Scaffold Underlying Temporal Integration
}

\author{
@Melanie M. Lee, ${ }^{1}{ }^{\circledR}$ Aristides B. Arrenberg, ${ }^{2}$ and ${ }^{-E m r e ~ R . F . ~ A k s a y ~}{ }^{1}$ \\ ${ }^{1}$ Institute for Computational Biomedicine and the Department of Physiology and Biophysics, Weill Cornell Medical College, New York, New York 10021, \\ and ${ }^{2}$ Developmental Biology, Institute Biology 1, Faculty of Biology and Center for Biological Signaling Studies, University of Freiburg, D-79104, Freiburg, \\ Germany
}

The accumulation and storage of information over time, temporal integration, is key to numerous behaviors. Many oculomotor tasks depend on integration of eye-velocity signals to eye-position commands, a transformation achieved by a hindbrain cell group termed the velocity-to-position neural integrator (VPNI). Although the VPNI's coding properties have been well characterized, its mechanism of function remains poorly understood because few links exist between neuronal activity, structure, and genotypic identity. To fill this gap, we used calcium imaging and single-cell electroporation during oculomotor behaviors to map VPNI neural activity in zebrafish onto a hindbrain scaffold consisting of alternating excitatory and inhibitory parasagittal stripes. Three distinct classes of VPNI cells were identified. One glutamatergic class was medially located along a stripe associated with the alx transcription factor; these cells had ipsilateral projections terminating near abducens motoneurons and collateralized extensively within the ipsilateral VPNI in a manner consistent with integration through recurrent excitation. A second glutamatergic class was more laterally located along a stripe associated with transcription factor $d b x 1 b$; these glutamatergic cells had contralateral projections collateralizing near abducens motoneurons, consistent with a role in disconjugate eye movements. A third class, immunohistochemically suggested to be GABAergic, was located primarily in the $d b x 1 b$ stripe and also had contralateral projections terminating near abducens motoneurons; these cells collateralized extensively in the dendritic field of contralateral VPNI neurons, consistent with a role in coordinating activity between functionally opposing populations. This mapping between VPNI activity, structure, and genotype may provide a blueprint for understanding the mechanisms governing temporal integration.

Key words: eye movements; microcircuit; neural integrator; patterning; persistent activity; temporal integration

\section{Introduction}

Temporal integration, the accumulation and storage of inputs over time, is the basis for many sensorimotor transformations and decision-making tasks. For example, temporal integration enables the maintenance of targeted eye position in the oculomotor system (Robinson, 1968), the memory of tactile stimulus frequency in the prefrontal cortex (Romo et al., 1999), and the storage of accumulated evidence in the lateral intraparietal area

Received July 23, 2014; revised April 9, 2015; accepted April 15, 2015.

Author contributions: M.M.L. and E.R.F.A. designed research; M.M.L. and A.B.A. performed research; M.M.L. and E.R.F.A. analyzed data; M.M.L., A.B.A., and E.R.F.A. wrote the paper.

This work was supported by the Tri-Institutional Computational Biology and Medicine Program to M.M.L.; the Deutsche Forschungsgemeinschaft DFG EXC307 and EXC294 to A.B.A.; and the Burroughs Wellcome Career Award at the Scientific Interface, a Searle Scholars Award, the Simons Foundation, National Science Foundation IIS1208088, and National Institutes of Health R01 EY021581 to E.R.F.A. We thank Robert Baker, Joseph Fetcho, and Mark Goldman for helpful comments on the manuscript; Charlotte Grove, Alex Ramirez, Kayvon Daie, and Sherika Sylvester for helpful discussions; the Joseph Fetcho laboratory for the vglut2 and glyt2 lines; Santanu Chakraborty for help with electroporation; and Lisa Schreiber for technical assistance.

The authors declare no competing financial interests.

Correspondence should be addressed to Dr. Emre R.F. Aksay, Institute for Computational Biomedicine and the Department of Physiology and Biophysics, Weill Cornell Medical College, 1300 York Avenue, New York, NY 10021. E-mail:ema2004@med.cornell.edu.

A. B. Arrenberg's present address: Werner Reichardt Centre for Integrative Neuroscience, University of Tübingen, Otfried-Müller-Str. 25, 72076, Tübingen, Germany.

DOI:10.1523/JNEUROSCI.3045-14.2015

Copyright $\odot 2015$ the authors $\quad 0270-6474 / 15 / 357903-18 \$ 15.00 / 0$
(Shadlen and Newsome, 2001). In all of these settings, the neural correlates to behavior are coordinated firing rate changes that persist long after termination of the presented stimulus. Although coding properties in these systems have been well studied, the mechanism of temporal integration is not fully understood. Elucidation of this mechanism requires understanding both the circuit-level organization within an integrating network and the relevant molecular and cellular properties of the constitutive neurons.

The functional properties of integration in the oculomotor system have been particularly well characterized. The oculomotor velocity-to-position neural integrator (VPNI) accumulates eye-velocity-encoded inputs to produce eye-position commands, serving as a common temporal integrator for many types of eye movements, including those controlled through the saccadic and optokinetic pathways shown in Figure $1 A$. The mechanism of integration in the VPNI is thought to depend critically upon positive feedback through recurrent network interactions (Robinson, 1989; Seung et al., 2000; Aksay et al., 2007; Fisher et al., 2013; Joshua et al., 2013). However, it has been difficult to connect this systems-level understanding with the anatomical and physiological properties of the neuronal elements in the circuit.

The recent identification in the larval zebrafish of a genotypic scaffold in the caudal hindbrain (Higashijima et al., 2004; 


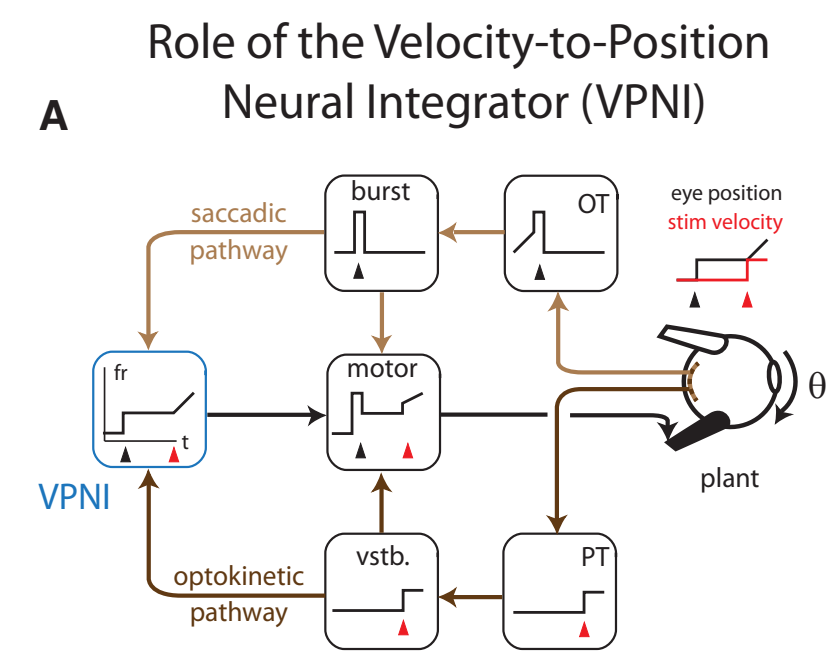

\section{How is the VPNI mapped onto the genotypic scaffold of the caudal hindbrain?}
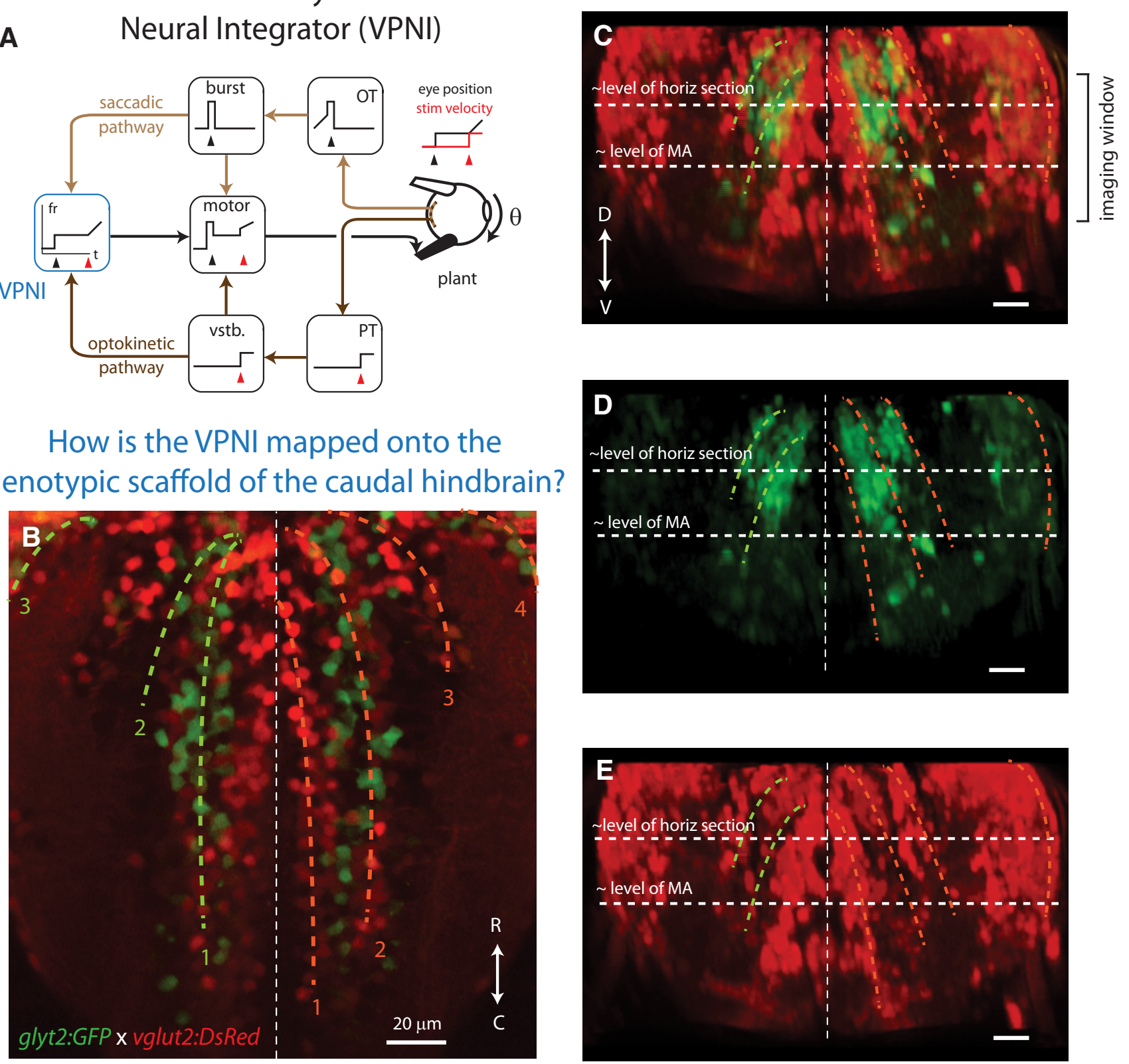

Figure 1. Linking coding of the VPNI with a structural and genotypic scaffold. $A$, Core oculomotor circuit mediating saccades and fixations (top, light brown) and optokinetic responses (bottom, dark brown). Adapted from Raphan and Cohen (1978) and Fuchs et al. (1985). In the saccadic pathway, the presentation of a visual target initiates a buildup of signal in the optic tectum (0T) (superior colliculus in mammals) at a location on a retinotopic map that encodes the angular displacement the eye must make to acquire the target. When this signal reaches a threshold (black arrowhead), a burst of activity is triggered in the saccade generator (burst). The burst signal is conveyed directly to the motor neurons (motor) to elicit a saccade that rapidly moves the eye onto the target. Because the transient burst signal is not able to maintain muscle contraction, signals from the saccade generator are also routed through the VPNI, which accumulates the burst input over time to generate an eye position-encoded step in firing rate (fr) that persists and provides a tonic drive to the motor neurons. In the optokinetic pathway, movement of a broad-field visual stimulus (red) generates a stimulus velocity signal in the pretectum (PT) that initiates an eye-velocity command signal in vestibular nuclei (vstb.). This velocity-encoded step is combined at the motoneurons with a position-encoded ramp generated by the VPNI to overcome the inertia of the plant and smoothly track movements in the visual field. $\boldsymbol{B}$, Horizontal section showing the genotypic scaffold in rhombomeres (rh) 7-8 of a $7 \mathrm{dpf} \mathrm{Tg}(\mathrm{vg} / \mathrm{lut2}$ :DsRed) x Tg(glyt2:GFP) fish. Red and green dashed lines indicate the approximate centers of the glutamatergic and glycinergic stripes, respectively. C, Coronal view of a stack of horizontal sections projected through rh 7-8 of a $7 \mathrm{dpf} \operatorname{Tg}(\mathrm{vg} / \mathrm{ut2}$ :DsRed) $x \operatorname{Tg}(g / \mathrm{yt2}: G F P)$ fish. White dashed lines indicate the approximate dorsoventral level of the horizontal section shown in $B$ and of the level in which the Mauthner axons (MA) are visible in rh 8 . Bracket at right indicates the dorsoventral range within which data were collected, $\sim 45 \mu \mathrm{m}$ above and $30 \mu \mathrm{m}$ below the MA level. $\boldsymbol{D}, \boldsymbol{E}$, Same coronal stack as $\boldsymbol{C}$, but spectrally separated to show (D) glyt2:GFP and (E) vglut2:DsRed expression.

Kinkhabwala et al., 2011; Koyama et al., 2011) where VPNI neurons are observed (Miri et al., 2011a) opens up the possibility of making such links. This scaffold consists of alternating stripes of glutamatergic and glycinergic cells arranged along approximately parasagittal planes (see Fig. $1 B-E$ ). Furthermore, particular stripes (or portions of stripes) are coextensive with transcription factor domains within which there is variation along the dorsoventral axis in age, axonal extent, and input resistance. An understanding of how the VPNI is distributed along this scaffold could lead to important clues as to the cellular and circuit mechanisms governing the process of temporal integration during oculomotor control. 
Here we mapped the VPNI onto this scaffold by combining calcium imaging during oculomotor behavior and single-cell electroporation in dual-transgenic zebrafish that express DsRed in glutamatergic neurons and GFP in glycinergic neurons. We identified three distinct classes of VPNI neurons based on neurotransmitter and morphological specification. When viewed in light of previous results (summarized by Fisher et al., 2013), these findings suggest distinct functional roles for each group, with one group important for hemifield-specific integration, a second for bilateral coordination, and a third for disconjugate control. Together, these findings represent an important step toward understanding the circuit-level structure-function relationships of the VPNI with molecular and cellular resolution.

\section{Materials and Methods}

Zebrafish preparation and dye loading. All experimental procedures were approved by the Institutional Animal Care and Use Committee of Weill Cornell Medical College. Physiological experiments were performed with 6-12 dpf zebrafish of either sex from two transgenic nacre mutant lines: $\operatorname{Tg}(\operatorname{slc17a6b}: D s R e d) n n s 9 T g$ (abbreviated as $\mathrm{Tg}($ vglut2:DsRed)) and $\mathrm{Tg}$ (slc17a6b:DsRed)nns9Tg $x \mathrm{Tg}$ (slc6a5:GFP)cf3/cf4TG (Tg(vglut2: DsRed) $x \operatorname{Tg}(g l y t 2: G F P)$ ) (McLean et al., 2007; Bae et al., 2009). Animals were reared at $28^{\circ} \mathrm{C}$ through day 5 and moved to room temperature at day 6 to slow the rate of development. Procedures for mounting of the larvae, preparation of calcium-sensitive dyes ( $5 \mathrm{~mm}$ Oregon Green 488 BAPTA-1 AM [OGB], Invitrogen O6807; or 2 mM rhod-2 AM [rhod 2], Invitrogen R1245MP), and loading of the dyes were similar to those previously described (Daie et al., 2015).

Two-photon calcium imaging. Synchronous two-photon calcium imaging and eye tracking were performed using a custom-designed system previously described (Daie et al., 2015). Functional imaging of calcium dynamics was performed at multiple planes in rhombomeres (rh) 7 and 8 of the caudal hindbrain, where VPNI neurons have been previously identified (Miri et al., 2011a). For each imaging plane, dorsoventral (DV) depth was measured relative to the level of the Mauthner axon (MA), which was identified in the contrast image of rh 8 , just above the inferior olive. This rh $8 \mathrm{MA}$ level was typically $20 \mu \mathrm{m}$ above the position of the Mauthner soma located in rh 4 . To position the imaging window $(\sim 184$ $\mu \mathrm{m}$ on edge) in rh 7 and 8 , we used the posterior otolith at the MA level as a guide because we had previously observed that at these ages its location was closely aligned with that of rh 5 (Miri et al., 2011a). The rostral edge of this imaging window was placed $\sim 45 \mu \mathrm{m}$ caudal of the center of the posterior otolith, thus aligning it with the border of rh 6 and 7 (Miri et al., 2011a). During subsequent analysis, slight corrections to this rostral border position were made by registering clearly identifiable anatomical features in the caudal hindbrain with those in control animals labeled with widely accepted rhombomeric indicators, as previously described (Daie et al., 2015). These anatomical features included the following: the rostrocaudal (RC) location of the point of maximal curvature in the lateral neuropil of rh 8 , the Ca reticulospinal neurons, a ventral glutamatergic cell population residing along the midline (see Fig. 2A, bottom), and myotome borders visible in contrast images.

In each fish, 3-7 horizontal planes were imaged for $\sim 10-15$ min per plane. Recordings at each plane included 5 min of spontaneous behavior in the light, $5 \mathrm{~min}$ with an optokinetic stimulus, and sometimes $5 \mathrm{~min}$ spontaneous behavior in the dark. During spontaneous behavior in the light, the fish was presented with alternating stationary dark and light stripes ( $1 \mathrm{~cm}$ wide, $1 \mathrm{~cm}$ tall) projected onto a $1 \times 6 \mathrm{~cm}$ piece of diffusion film (Novatron 4008) placed 1-2 cm in front of the fish. For the optokinetic stimulus, the velocity of the stripes moved sinusoidally, reaching a peak velocity of $\sim 30 \mathrm{deg} / \mathrm{s}(\sim 1 \mathrm{bar} / \mathrm{s})$.

Functional calcium imaging for all behavioral conditions was performed with the excitation wavelength set to $790 \mathrm{~nm}$ and powers of $10-15 \mathrm{~mW}$. Immediately following functional imaging, reference images of XFP expression (DsRed, GFP) in the same plane were collected with excitation wavelength set to 930 or $950 \mathrm{~nm}$ and power adjusted to $15-20$ $\mathrm{mW}$. All image time series were collected at $\sim 1 \mathrm{~Hz}$ and images were $512 \times 512$ pixels.
Single-cell electroporation. Single-cell electroporation with a fluorescent indicator of VPNI and candidate VPNI neurons was performed to examine cell morphology. Larvae were prepared for imaging as described above, but additionally a small perforation was made in the skin at the caudal end of the cerebellum to facilitate penetration of an electroporation electrode. The perforation was made using a sharp glass electrode coated with a saturated solution of DiI dissolved in DMSO (DiI, Invitrogen D7777) while the fish was anesthetized with $50 \mathrm{mg} / \mathrm{L}$ MS-222. Sharp pipettes $(\sim 15-20 \mathrm{M} \Omega$ in Evans solution, borosilicate, $1.0 \mathrm{~mm} \mathrm{OD}, 0.58$ mm ID, P-30 puller, Sutter) were back-filled with 8 mm Alexa-488 dextran (Invitrogen D22910, dissolved in Evans solution) or 10 mм Texas Red Dextran (Invitrogen D3329, dissolved in Milli-Q water). In some cases, the glass pipettes were first front-filled with $1.7 \%$ low-melting point agarose to enable consistent iontophoresis and minimize spurious cell fills. In other cases, to prevent front-filling and subsequent dilution of the dye solution, continuous back pressure of $<1$ psi was applied as the electrode was advanced to the perforation and was immediately turned off upon entry into the brain (Picospritzer III, Parker Hannifin; micromanipulator MP-285, Sutter). The electrode was then advanced lateral and caudal of a target cell before being retracted diagonally at least $\sim 30$ $\mu \mathrm{m}$ (or 3-6 cell diameters) to clear the tip of possible obstruction by tissue debris, then advanced horizontally and vertically toward the cell of interest. The electrode tip was positioned so that it made close contact with the cell membrane, seen optically as a slight distortion of the cell silhouette; then a voltage pulse train was applied to transiently porate the cell membrane and deliver the positively charged dye solution. A 2-3 V pulse train consisting of $2 \mathrm{~ms}$ square pulses delivered at $200 \mathrm{~Hz}$ was generated using a DAQ card (PCI 6110, National Instruments) and a linear stimulus isolator (WPI A395) and was delivered for $0.5 \mathrm{~s}$ via a microelectrode amplifier (AM Systems 1800) in stimulation mode. Typically, the cell soma and proximal dendrites filled within a minute of applying the pulse train. Imaging was performed both within minutes of electroporation and $30 \mathrm{~min}$ to a day later after waiting for axonal projections to completely fill.

Immunohistochemistry. All experimental procedures for immunohistochemical analysis conformed to the institutional guidelines of the University of Freiburg and the local government (Regierungspräsidium Freiburg). Immunohistochemistry was performed on $\mathrm{Tg}(\mathrm{slc17a6b}$ : EGFP)zf139Tg ( $\mathrm{Tg}($ vglut2:GFP $)$ ) transgenic animals. $\mathrm{Tg}$ (vglut2:GFP) larvae were immunostained using the primary antibodies rabbit anti-GABA (1:500, Sigma A2052), chicken anti-GFP (1:400, Invitrogen A10262), and the secondary antibodies anti-rabbit Alexa-546 and anti-chicken Alexa488 (1:1000, Invitrogen). The whole-mount immunostained larvae were imaged using a spinning disk confocal microscope. The images shown in Figure $5 A-C$ are average projections across $\sim 5 \mu \mathrm{m}$ in the DV axis.

Analysis of cell fluorescence activity. All analysis of image time series was completed in MATLAB (The MathWorks). Custom scripts were developed for motion correction, functional identification of cells, calculation of cellular fluorescence response, and alignment of fluorescence time series to correct for temporal undersampling relative to eye tracking data.

An iterative algorithm for subpixel image registration by crosscorrelation (Guizar-Sicairos et al., 2008) was first applied to all functional imaging data to remove erroneous signals introduced by in-plane motion. This algorithm involved aligning individual frames with a reference image generated by taking the time-projection (average) across all frames. An image time series with significant in-plane motion would have a relatively blurry reference image before alignment. Frames for which the rate of shift exceeded a threshold of $0.15 \mu \mathrm{m} /$ frame were excluded from analysis, and the remaining aligned frames were timeaveraged to produce an updated reference image. Sequential iterations of these steps were applied until all frames were aligned to within the shift rate threshold; this served to simultaneously improve the sharpness of the reference image and the frames that were aligned to it.

Following motion correction, correlation maps were generated for each dataset to identify eye movement-related neurons (Miri et al., 2011a, 2011b). These correlation maps describe, at pixel-by-pixel resolution, the temporal correlation of calcium concentration changes with simultaneously measured eye and stimulus position and velocity variables. Hemifield-specific eye and stimulus position and velocity were 
mapped to variables corresponding to associated models of fluorescence reported by a calcium indicator by convolving the behavioral variables with a calcium impulse response function (CIRF). The CIRF takes into account the slow intracellular calcium buffering and clearance associated with a cell's action potential. The CIRF is characterized by its time constant of decay, measured across OGB-loaded VPNI cells to have a population median of $1.9 \mathrm{~s}$ (Daie et al., 2015). Fluorescence time series of image pixels were then linearly regressed against these CIRF-convolved behavioral variables, and a correlation map was generated from the regression coefficients. ROIs corresponding to candidate eye movementrelated neurons were selected for data acquired first during saccades and fixations through a user interface that presented both the correlation map and a time-averaged reference frame of the fluorescence activity. Next, these ROIs were copied onto the time-projection image of fluorescence activity acquired during optokinetic stimulation, and aligned to the new image by determining the $2 \mathrm{D}$ cross-correlation between the time-averaged fluorescence images of the two behavioral conditions (MATLAB function normxcorr2). Additional ROIs were drawn on the second correlation map if more eye movement-related cells were found and their coordinates were reverse-aligned to the first behavioral condition using the same offset but with a reversed sign. Average shift values in the RC and mediolateral (ML) dimensions were $-0.12 \pm$ 0.61 and $-1.5 \pm 1.9 \mu \mathrm{m}$, respectively ( $n=38$ planes). This procedure ensured that all possible eye movement-related neurons were identified and aligned across the datasets corresponding to the two behavioral conditions.

Fluorescence time series were then calculated for each ROI by computing the average fluorescence within each ROI for each frame and then converting it to a percentage change in fluorescence by taking the difference of each trace from the average fluorescence and then dividing by the average. To facilitate analysis of relationships between neural activity and eye movements, fluorescence time series were interpolated with a time step of $50 \mathrm{~ms}$. They were then truncated to begin at the start time of the last acquired ROI in an imaging frame, and end at the earliest end time across fluorescence, eye, and stimulus time series. The fluorescence time series were additionally detrended using a baseline correction procedure in which a quadratic fit to the bottom $20 \%$ of points was subtracted from each time series. Finally, intervals containing body-movement related artifacts that were not eliminated by the motion correction algorithm were excluded by hand from further analysis.

Functional classification of cells. Cells were classified as VPNIs based on the correlation of their full ROI fluorescence time series with eye and/or stimulus variables. These correlations were used to define separate maximal behavioral-sensitivity measures during spontaneous fixations and optokinetic responses, and subsequently these measures were used to set criterion for inclusion in the analyzed VPNI population. For spontaneous activity, the maximum behavioral sensitivity for a cell was defined as the greater of the correlation of its fluorescence time series with CIRFconvolved ipsiversive (a) eye position and (b) eye velocity. For optokinetic response, the maximum sensitivity was defined as the greater of the correlation of its fluorescence time series with CIRF-convolved (a) stimulus position and (b) stimulus velocity. Cells were then classified as VPNI if they satisfied two conditions: (1) the maximum sensitivity during spontaneous behavior was at least 0.4 , and (2) the ratio of maximum sensitivity during spontaneous behavior to maximum sensitivity during optokinetic behavior was no greater than 3 . Condition 1 excluded vestibular and velocity-sensitive neurons, which in teleost fish do not respond during spontaneous eye movements (Beck et al., 2006); and Condition 2 excluded saccadic burst neurons, which do not show responses correlated with slow-phase optokinetic movements (Scudder et al., 2002).

Separating OGB, DsRed, and GFP signals. To determine colocalization of functionally specified ROI with the XFPs (DsRed or GFP), it was necessary to first separate fluorescence signals from DsRed, GFP, and OGB. Separation of the XFPs from OGB was possible because of the unimodal excitation spectra of the two XFPs (with GFP peaking 930$950 \mathrm{~nm}$ and DsRed increasing monotonically from 800 to $1000 \mathrm{~nm}$ ) and the bimodal excitation spectrum of OGB (with peaks at 790 and $930 \mathrm{~nm}$ ) (Brondi et al., 2012).
We imaged calcium activity at $790 \mathrm{~nm}$, where OGB signals were dominant, and the XFPs at $930 \mathrm{~nm}$, where both XFPs and OGB were present. Before separating the fluorescence signals, we subtracted from the timeprojection images at 930 and $790 \mathrm{~nm}$ contributions from background noise, and then aligned the images. Noise was assessed by taking the mean of pixel intensities at each wavelength within an ROI outside of the brain. Following noise subtraction, the time-projection image at $930 \mathrm{~nm}$ was registered with the time-projection image at $790 \mathrm{~nm}$ to correct any inplane shifts that may have occurred between the acquisitions at the two wavelengths. Registration was done by hand-selecting at least two ROI from each plane at each wavelength and aligning their centroids. ROIs were selected in the green channel time-projection images, where OGB was present at both wavelengths.

To separate the XFPs from OGB, we used the fact that, for each wavelength, $\lambda \epsilon\{790,930\}$ and channel $k \in\{R, G\}$, the time-averaged fluorescence image could be represented as the linear combination of contributions from the XFP and OGB fluorophores as follows:

$$
\bar{F}_{\lambda, k}=F_{\lambda, k}^{o g b}+F_{\lambda, k}^{d s r e d}+F_{\lambda, k}^{g f p}
$$

where the bar denotes a measured/observed quantity; and that the fluorescence of OGB at the two wavelengths and in the two channels could be related simply by scale factors:

$$
\begin{aligned}
& F_{930, G}^{o g b}=a_{G} \cdot F_{790, G}^{o g b} \\
& F_{930, R}^{o g b}=a_{R} \cdot F_{790, G}^{o g b}
\end{aligned}
$$

Given that, at $790 \mathrm{~nm}$, the intensity of OGB is 15 -fold greater than that of either XFP (Drobizhev et al., 2009; Mütze et al., 2012), we assumed that the contributions of the XFPs at $790 \mathrm{~nm}$ are negligible. The signals resulting only from the XFPs at $930 \mathrm{~nm}\left(F_{930, k}^{x f p}\right)$ could then be determined as follows:

$$
F_{930, k}^{d s r e d}+F_{930, k}^{g f p} \approx \bar{F}_{930, k}-a_{k} \cdot \bar{F}_{790, G}
$$

To determine the scale factors $a_{k}$, we next examined for different planes histograms of the OGB-subtracted pixel intensities from all of the ROIs for functionally identified cells. We noted that, for each plane, regardless of the value of $a_{k}$, this histogram had two or three peaks, with the peak associated with the lowest fluorescence intensities necessarily corresponding to those cells that were XFP-negative. Because we had previously subtracted from these images an average noise intensity, we reasoned that, when the value of the scale factor $a_{k}$ is appropriately chosen, this peak value should be at zero intensity. Thus for each plane, the scale factors $a_{k}$ were sampled at uniform intervals within the ranges $a_{R} \epsilon[0.02,0.4]$ and $a_{G} \epsilon[1.3,3.0]$ to determine the value for that plane that resulted in a first-peak location at the origin.

Next, it was necessary to demix the fluorescence signals from DsRed and GFP because both fluorophores contributed signals in both the red and green channels (Bagwell and Adams, 1993). Their contributions could be related by the following:

$$
\begin{aligned}
& F_{930, R}^{d s r e d}+\alpha \cdot F_{930, G}^{g f p}=F_{930, R}^{x f p} \\
& \beta \cdot F_{930, R}^{d s r e d}+F_{930, G}^{g f p}=F_{930, G}^{x f p}
\end{aligned}
$$

where

$$
\begin{aligned}
& \alpha=F_{930, R}^{g f p} / F_{930, G}^{g f p}=\alpha_{0} \cdot\left(g_{R} / g_{G}\right) \\
& \beta=F_{930, G}^{d s r d} / F_{930, R}^{d s r e d}=\beta_{0} \cdot\left(g_{G} / g_{R}\right)
\end{aligned}
$$

$\alpha_{0}$ and $\beta_{0}$ are constants describing the ratios of the integrated red and green fluorescence, and the variables $g_{R}$ and $g_{G}$ denote detection gains. The constants $\alpha_{0}$ and $\beta_{0}$ were determined from published in vitro fluorescence emission spectra for GFP (Heim et al., 1995) and mature DsRed (Baird et al., 2000); the resulting values were $\alpha_{0}=$ 0.094 and $\beta_{0}=0.032$. Because the detection gains vary with depth in the brain, we determined the gain ratios empirically for each plane by solving Equations 4 and 5. In theory, either GFP-positive or DsRedpositive cells can be used to calculate the fluorescence ratios in Equation 5. However, because DsRed takes a long time to mature and is green in its immature form (Baird et al., 2000), we solved for Equation 
5 using hand-selected easily identified GFP-positive cells (average of 5 cells per plane) for which this variation in color does not occur; for these cells, $F_{930, R}^{d s r e d}=0$ and; therefore, $g_{R} / g_{G}=F_{930, R}^{x f p} /\left(\alpha_{0} \cdot F_{930, G}^{x f p}\right)$. The resulting scale factors obtained across all planes varied between $\alpha \epsilon[0.01,0.07]$ and $\beta \epsilon[0.04,0.02]$. Finally, XFP-specific images for each plane were determined from the following:

$$
\begin{aligned}
& F_{930, R}^{d s r e d}=\left(\frac{1}{1-\alpha \cdot \beta}\right) \cdot F_{930, R}^{x f p}-\left(\frac{\alpha}{1-\alpha \cdot \beta}\right) \cdot F_{930, G}^{x f p} \\
& F_{930, G}^{g f p}=\left(\frac{1}{1-\alpha \cdot \beta}\right) \cdot F_{930, G}^{x f p}-\left(\frac{\beta}{1-\alpha \cdot \beta}\right) \cdot F_{930, R}^{x f p}
\end{aligned}
$$

The procedure for registering and separating XFP and OGB timeprojection images was also applied to data collected from fish containing only the transgene vglut2:DsRed.

Identifying glutamatergic or glycinergic VPNI neurons. Next, to identify the neurotransmitter phenotype of the functionally specified VPNI neurons, we manually categorized VPNI neurons as being XFP-positive, XFP-negative, or indeterminate. A DsRed-positive cell was glutamatergic, and a GFP-positive cell was glycinergic; XFP-negative cells were either negative due to mosaicism or potentially of a different neurotransmitter phenotype; those cells that could not be confidently categorized as either XFP-positive or XFP-negative were removed from the analyzed pool. To categorize the cells, we used a computer interface that enabled simultaneous viewing of the VPNI ROI on the time-projection XFP-specific image $\left(F_{930, R}^{d s r e d}, F_{930, G}^{g f p}\right.$; Eq. 6), the images of OGB-loaded cells $\left(\bar{F}_{790, G}\right)$, and the corresponding correlation maps for the two behavioral conditions (see Analysis of cell fluorescence activity). Cells were first categorized independently by two separate users; the results from the two independent evaluations agreed by $85 \%$ ( 314 of 371 cells). The $15 \%$ of cells ( 57 of 371 cells) categorized differently were then evaluated by both users together. Of these, a consensus could be reached for $91 \%$ ( 52 of 57 cells), with $58 \%$ of them being placed in an indeterminate pool and discarded from further analysis ( 30 of 52 cells). The final dataset consisted of 86 XFP-positive and 247 XFP-negative VPNI cells. Among the XFP-positive group, 81 cells were selected in the first independent round of evaluations and 5 were additionally agreed upon during the jointly performed selection. Among the XFP-negative group, 230 cells were selected in the first independent round, and 17 were subsequently agreed upon and added during the joint evaluation.

Finally, to check that the categorization procedure was done properly, we evaluated two parameters that quantified our qualitative observations that XFP-positive and -negative cells differed overall in the following: (1) their mean within-ROI XFP-fluorescence intensity and (2) the ratio of within-ROI to surround intensities. First, to correct for intensity variations within each plane, we segmented each plane into four quadrants within which pixel intensities were normalized to the interval $[0,1]$. To quantify the observation that XFP-positive cells tend to have higher average XFP-pixel intensities, we compared the mean ROI intensity of XFP-positive and -negative cells. This mean ROI intensity parameter differed significantly between the XFP-positive and -negative populations $\left(p=1.67 \times 10^{-33}\right.$; rank sum test), with XFP-positive and -negative cells having mean intensities of $0.56 \pm 0.48(\mathrm{SD})$ and $0.091 \pm 0.12$, respectively. Second, the relative intensity of an ROI with respect to its surround was measured by comparing the distribution and mean of pixel intensities within an annulus about the ROI and within the ROI itself. The mean ROI-to-annulus intensity parameter also differed significantly between the XFP-positive and -negative groups $\left(p=8.0 \times 10^{-37}\right.$; rank sum), with XFP-positive and -negative cells having mean ROI-toannulus intensity ratios of $1.45 \pm 0.59$ and $0.41 \pm 0.33$, respectively.

Stripe identification and localization. To quantitatively examine how cells were distributed along the glutamatergic stripes, the time-projection image of XFP fluorescence for each plane was digitally segmented into 4 stripe regions per side of the brain as follows. First, the midline was defined as the line joining two user-identified points along the midline. Next, a border was drawn by a user-interface to delineate the first (medial) and second glutamatergic stripes on one side of the brain. This border could be seen as a gap between the two most medial columns of cells. When this gap was sizable, the border was drawn so that it fell just lateral of the more medial stripe. This gap was present at all dorsalventral levels but was most prominent in planes ventral of the MA level. Above the MA level, the first glycinergic stripe (when present in the dual-transgenic fish) was sometimes used as a guide to separate the first two glutamatergic stripes, but the two stripes were still sufficiently separated in the absence of the glycinergic stripe, except at the rostral edge of the imaging window ( $\mathrm{rh} 6 / 7$ border) where the stripes converged. The border was then interpolated with a smoothing spline (MATLAB function csaps) and reflected over the midline to define the corresponding border on the contralateral side. Borders were drawn in a similar way between glutamatergic stripes 2 and 3, 3 and 4, and at the lateral edge of the hindbrain. To confirm continuity of the stripes across planes in each fish, the coordinates for the borders were collected into a 3D stack and compared with a reference 3D stack of borders drawn similarly, but at high DV resolution that enabled clear visualization of the stripes from a coronal view (compare Fig. 1C). A similar procedure was applied to identify stripe peaks/centers.

To validate that the segmentation procedure resulted in appropriate stripe regions and to quantify the actual stripe population distribution in the brain (see Fig. 3B), we made histograms across normalized ML distance. For each cell, we calculated its ML position and then normalized it by the distance between the stripe center and border. This normalized distance was then multiplied by the mean distance of the stripe center from the midline. This normalization accounted for the fact that the stripes do not run precisely parallel to the midline and allowed us to determine whether the stripe distribution was multimodal along the ML axis or was rather a flat, homogeneous distribution.

Cell reconstructions. $3 \mathrm{D}$ reconstructions of single neuron fills were performed using the Simple Neurite Tracer plug-in in ImageJ (Longair et al., 2011).

Estimation of neuronal persistence. Neuronal persistence for a given cell during spontaneous behavior was calculated from its saccade-triggered averaged fluorescence response (STAF) in three steps. First, the STAF was deconvolved to obtain the cell's estimated firing rate. Second, the deconvolved fluorescence was fit to a model to determine the decay time, $\tau$, of the estimated firing rate during the fixation period. Third, the fit was normalized and neuronal persistence, $\rho$, was quantified as the area under the normalized fit. These steps are detailed in the following.

The estimation of a cell's firing rate from its fluorescence response was based on a modification of previously described methods (Miri et al., 2011b). Briefly, we calculated the saccade triggered average of the cell's fluorescence response (STAF) by averaging over a minimum of 3 saccades preceding fixations that lasted at least $7 \mathrm{~s}$. The STAF was filtered with an adaptive cubic spline that fit the fluorescence in the interval $2 \mathrm{~s}$ before and $1 \mathrm{~s}$ after saccade onset with high fidelity, and produced a smoothed approximation of fluorescence for the remainder of the fixation period. The filtered fluorescence was then deconvolved with the CIRF (see Analysis of cell fluorescence activity) to obtain an estimation of firing rate.

The estimated firing rate (deconvolved STAF) was next fit to a model of the form $r(t)=a \cdot e^{-t / \tau}+c$. The fit was performed over a $5 \mathrm{~s}$ interval, beginning $1 \mathrm{~s}$ after the saccade and ending $6 \mathrm{~s}$ after the saccade, to estimate the parameters $a$ and $\tau$. The MATLAB function fit was used, with $a$ constrained to be non-negative and $\tau$ seeded to two initial values, 1 and -1 . The firing rate null position parameter, $c$, was held fixed during the fit and was estimated by taking either the upper $(c u b)$, lower bound $(\mathrm{clb})$, or middle ( $\mathrm{cmid}$ ) of the firing rate null range determined from the deconvolved ipsiversive and contraversive STAF (Miri et al., 2011b). Each cell was fit 6 times, corresponding to 3 null values $c$ and two different $\tau$ seeds, and the best fit was selected for the third step of analysis (described in the next paragraph). The quality of the fits was assessed by calculating the coefficient of determination $\left(R^{2}\right)$ of an augmented regression, designed to capture the goodness of fit of the area under the firing rate curve. This adjusted $R^{2}$ was defined as the concatenation of the fit of the ipsiversive firing rate and the same fit but reflected over the horizontal axis (therefore identical firing rate and fit, but reversed in sign) as it better captured fit quality of both the amplitude and decay time of the exponential. Only qualifying fits $\left(R^{2}>0.9\right.$, determined empirically) were included in subsequent analysis and statistics. 
To calculate the persistence measure $\rho$, the fit to the estimated firing rate was normalized so that the peak firing rate during the first $0.5 \mathrm{~s}$ of the fixation period was set to $1 . \rho$ was then estimated as the cell's average response during the same $5 \mathrm{~s}$ fixation interval as used above; this average is given by $\frac{1}{T} \sum_{t} r_{t}$, where $T$ is the number of data points during the fixation and $r_{t}$ is the normalized firing rate response at time $t$. With this measure, a perfectly persistent cell would have $\rho=1$ and a cell without persistent firing would have $\rho=0$. Cells for which $\rho>1$ would have firing rates that continue to rise after the initial $0.5 \mathrm{~s}$ of the fixation period, corresponding to an unstable cell with negative $\tau$. Cells for which $\rho>2$ were found to be noisy and excluded from further analysis $(\sim 1.5 \%$ of VPNI cells).

\section{Results}

Here we mapped the VPNI onto a structural and genotypic scaffold in the zebrafish hindbrain, enabling us to link VPNI coding properties with the cellular and molecular properties of the circuit's constitutive neurons. The hindbrain of the larval zebrafish is divided along the rostrocaudal (RC) axis into eight segments called rhombomeres (rh). Localized ablation and halorhodopsinmediated optical inactivation of the caudal hindbrain have demonstrated that rh 7 and 8 are necessary for temporal integration during oculomotor control (Miri et al., 2011a, 2011b). Cells in the hindbrain are further organized into seven approximately parasagittal planes of cells that alternate between excitatory and inhibitory cell type (Higashijima et al., 2004; Kinkhabwala et al., 2011; Koyama et al., 2011). These stripes are associated with transcription factors that specify some of the neuronal characteristics (including morphological features) that the cells acquire in development (Lewis, 2006). In particular (and in mediolateral (ML) order), most, if not all, first glutamatergic stripe neurons express alx (chx10 in mammals), first glycinergic stripe neurons express engrailed-1, and second glutamatergic and second glycinergic stripe neurons express $d b x 1 b$ (Kinkhabwala et al., 2011). In the following, we used calcium imaging to determine how the VPNI is distributed onto this hindbrain scaffold. We also used targeted electroporation of single cells to help identify the key morphological features of the different VPNI cell classes. Finally, we revealed links between VPNI functional properties specified by a neuronal persistence measure and the structural-genotypic organization of the VPNI. Together, these results provide links to moleculargenetic factors that specify anatomical and physiological properties of cells in the VPNI, shedding insight into the mechanism of integration and potentially serving as a technological lever for further studies that require restricted expression in the oculomotor integrator.

\section{Spatial distribution of the VPNI on a hindbrain genotypic scaffold}

We first mapped the VPNI using two-photon calcium imaging of rhombomeres 7 and 8 in a dual-transgenic zebrafish line where glutamatergic neurons expressed DsRed, and glycinergic neurons expressed GFP (vglut2:DsRed, glyt2:GFP) ( $n=5$ fish). Additional mapping data were acquired in a single transgenic vglut2:DsRed line alone $(n=2)$. Neuronal activity was measured during two distinct oculomotor behaviors, spontaneous saccades and fixations, and optokinetic tracking (OKR), both of which engage the VPNI. During spontaneous behavior, zebrafish were presented with a stationary stimulus consisting of a pattern of alternating dark and light stripes. Zebrafish typically performed a sequence of saccades, usually in alternating directions to an extreme eye position, with fixations lasting up to tens of seconds. On average, spontaneous eye movements spanned a range of $11.9 \pm 5.8^{\circ}$ (mean $\pm \mathrm{SD})$, with mean and median fixation times of $17.1 \pm$ 10.8 and $12.3 \mathrm{~s}$, respectively ( $n=7$ fish). During optokinetic tracking, the stimulus pattern moved with a velocity that varied sinusoidally at a frequency of $0.067 \mathrm{~Hz}$, reaching a peak velocity of $\sim 30 \%$. The corresponding range of eye movements was $14.7 \pm 6.9^{\circ}$; zebrafish also performed fast-phase eye movements with an average interval of $15.4 \pm 14.5 \mathrm{~s}$ (median $11.4 \mathrm{~s}$ ). In both behavioral conditions, the two eyes generally moved in a conjugate manner.

VPNI activity was imaged at 3-7 horizontal planes per fish after bolus loading of the calcium-sensitive dye OGB $(n=371$ cells across both single- and dual-transgenic animals). Imaging was performed within a dorsoventral (DV) window that spanned from $45 \mu \mathrm{m}$ above to $30 \mu \mathrm{m}$ below the level where the MA was visible in rh 8 (Fig. 1C). For a given fish, we imaged each plane for at least $10 \mathrm{~min}$ : first for $5 \mathrm{~min}$ of spontaneous behavior in the light and then for $5 \mathrm{~min}$ of optokinetic reflex behavior. OGB signal was distinguished from DsRed and GFP (together referred to as XFP) signals by comparing images acquired at two excitation wavelengths (see Materials and Methods). VPNI neurons were identified by using a correlation map generated by regressing the fluorescence activity of individual pixels against eye movement regressors generated by convolving hemifield-specific eye position and velocity traces with a CIRF describing the relationship between VPNI firing rates and calcium dynamics (see Materials and Methods) (Miri et al., 2011a).

Typical activity patterns for VPNI cells are seen in Figure 2. Data are shown for two planes: one $11 \mu \mathrm{m}$ above the average level of the MAs $\left(^{*}\right)$ in the caudal hindbrain (top) and the other $21 \mu \mathrm{m}$ below (bottom). During spontaneous behavior, the fluorescence of VPNI cells (Fig. 2A, white outlines) exhibited relatively sharp increases with saccades in the ipsilateral direction (Fig. 2B; e.g., the activity of cells 1-3 increases with eye movements to the left [dotted gray vertical lines], and the activity of cells 4-6 increases with eye movements to the right [dashed gray vertical lines]). During fixations, cells maintained sustained changes in fluorescence, but with some variability between cells in the rate of relaxation to baseline (compare cells 4 and 5). During optokinetic behavior (Fig. 2C), the fluorescence of VPNI cells generally varied with stimulus position (e.g., cells 2 and 9; solid gray vertical lines indicate the slow-phase peaks of eye position in the left movement field), whereas the responses of a minority aligned better with the velocity of the stimulus (e.g., compare cell 10 with the dashed black stimulus velocity trace). Additionally, different cells exhibited different degrees of saccade sensitivity during the fast phase (compare cells 4 and 6). For the population, correlations to eye position and eye velocity during spontaneous behavior were $0.47 \pm 0.19$ and $0.29 \pm 0.19$, and correlations during optokinetic responses were $0.47 \pm 0.23$ and $0.27 \pm 0.17$.

In general, VPNI neurons appeared to be broadly distributed across the hindbrain scaffold. In Figure $2 A$, red and green dashed lines are used to indicate approximate locations of glutamatergic and glycinergic stripes, which were visually identified based on continuity across all imaged planes (see Materials and Methods). VPNI neurons were found along the first (e.g., cells 1-4), second (e.g., cells 8-10), and third (e.g., cells 5, 6, 11, 12) glutamatergic stripes, and some of these cells were positive for DsRed expression (e.g., cells 1, 2, 4). Cells were also distributed along the first and second glycinergic stripes (data not shown), but with relatively few cells positive for GFP expression. In ventral planes (bottom), many neurons negative for both fluorescent tags were located lateral of the 


\section{Functional Identification of VPNI Neurons using Calcium Imaging}

A
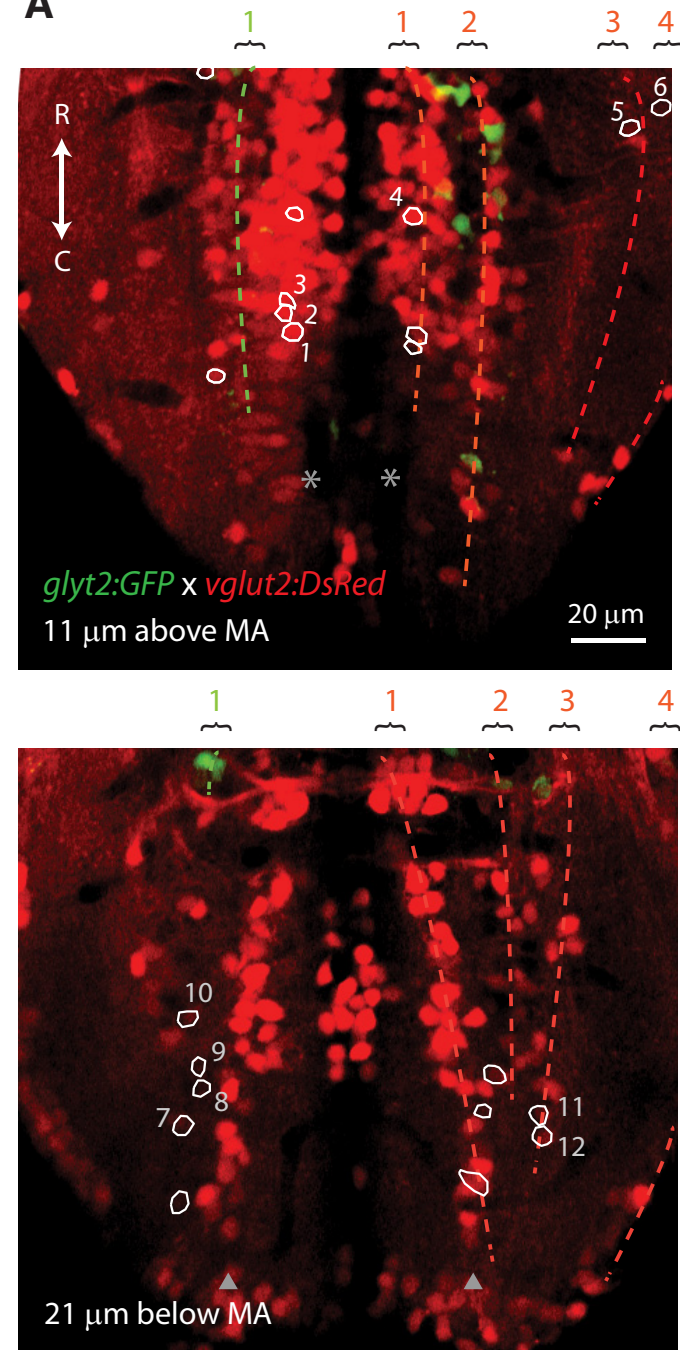

B

Spontaneous
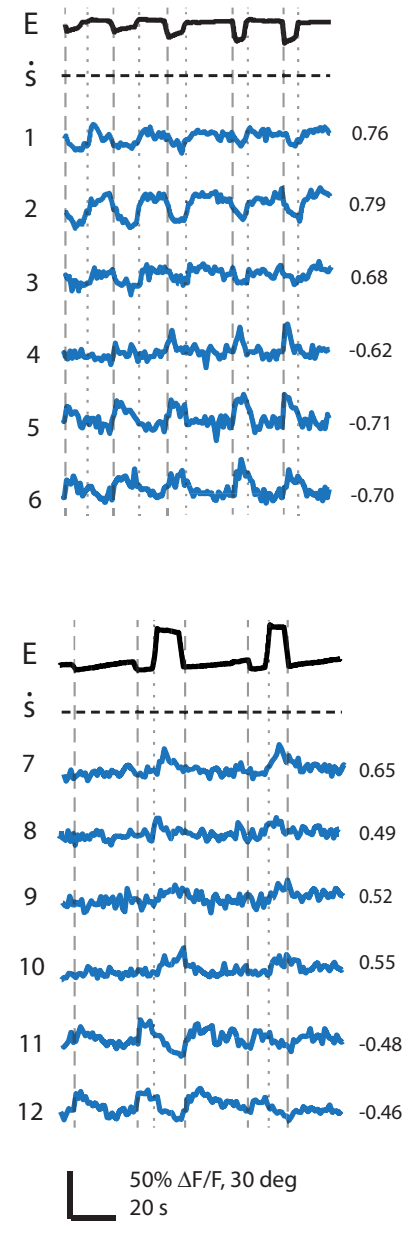

C Optokinetic
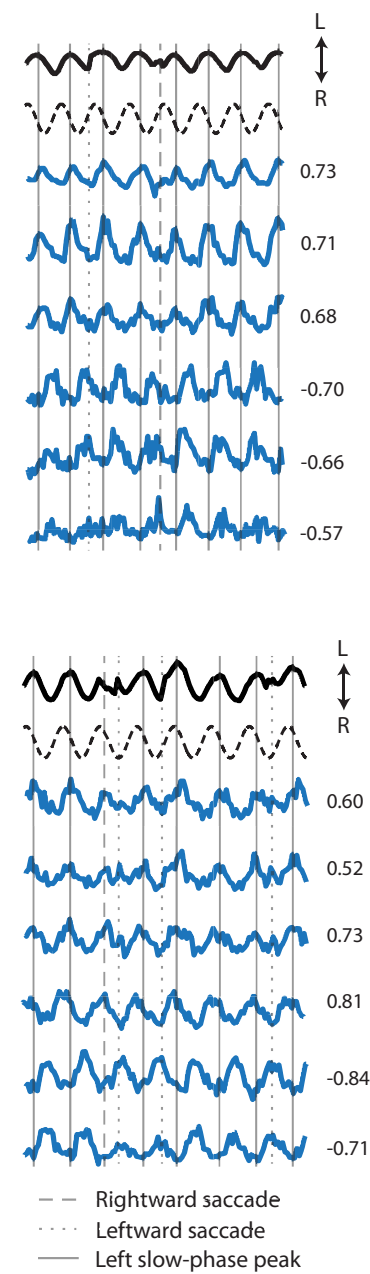

Figure 2. Spatial distribution of VPNI cells on the genotypic scaffold and dynamics during optokinetic and spontaneous response. $A$, Horizontal view of rh 7-8 in the $T g(v g / u t 2: D s R e d) x$ Tg(glyt2:GFP) line at two different DV depths. White ROIs indicate locations of VPNI cells; numbered ROls correspond to cells whose activities are plotted in $\boldsymbol{B}$ and $\boldsymbol{C}$. Red and green numbers at top indicate glutamatergic (red) and glycinergic (green) stripe peaks. Top, asterisks: MAs. Bottom, triangles: inferior olive. B, C, Eye position (black), stimulus velocity (dashed black), and fluorescence time series (blue) during spontaneous saccades and fixations $(\boldsymbol{B})$ and optokinetic response ( $\boldsymbol{C}$. Numbers to the left of traces correspond to ROI numbers in $\boldsymbol{A}$, and numbers to the right are the cells' maximum correlation to eye position $(\boldsymbol{B})$ or optokinetic stimulus position $(\boldsymbol{C})$. $\boldsymbol{B}, \boldsymbol{C}$, Light gray dotted and dashed vertical lines indicate approximate times of leftward and rightward saccades, respectively. $\boldsymbol{C}$, Solid gray vertical lines indicate approximate peaks of slow-phase eye position in the left movement field.

first glutamatergic stripe and comingled with the second glutamatergic stripe (e.g., cells 9 and 10).

To quantify these observations, we first determined in the dual-transgenic dataset $(n=273)$ what fractions of VPNI cells were glutamatergic, glycinergic, and potentially of a different neurotransmitter type (see Materials and Methods). Here, and in the rest of the results, data from fish of different ages were grouped together as we saw no significant difference when we segregated the data by age (data not shown). Of the 245 neurons that could be confidently categorized, $25 \%$ ( 61 of 245 cells) were glutamatergic, only $2 \%$ of the population ( 5 of 245 cells) were glycinergic, and 73\% (179 of 245 cells) of VPNI cells were XFPnegative (Fig. $3 A$ ). To understand how the relative abundance of glutamatergic and glycinergic cells compared with what could be expected by chance, we assessed the prevalence of DsRed-positive to GFP-positive cells in dual-transgenic fish by counting, independent of function, all XFP-positive cells in rhombomeres $7-8$.
This showed that, in general, DsRed-positive cells were four times more prevalent than GFP-positive ones. The greater number of DsRed-positive cells, however, does not account for the 13-fold greater percentage of VPNI cells found to be glutamatergic compared with those identified as glycinergic $\left(p=5.8 \times 10^{-4}, z\right.$-test for two proportions). These data thus suggest that, in the caudal hindbrain of the larval zebrafish: (1) there is a sizable of population of glutamatergic VPNI neurons; (2) glycinergic neurons do not contribute significantly to the VPNI; and (3) it is likely that there is one or more additional classes of VPNI neurons.

Next, we examined the distribution of VPNI cells in the DsRed-positive subgroup relative to the glutamatergic stripes. We now also included data that we had collected under identical conditions from fish only expressing the vglut2:DsRed transgene; given the negligible percentage of glycinergic VPNI neurons identified above, neurons that did not express DsRed in this single transgenic line were merged with the pool of DsRed-negative 


\section{Distribution of VPNI cells relative to glumatergic stripes}
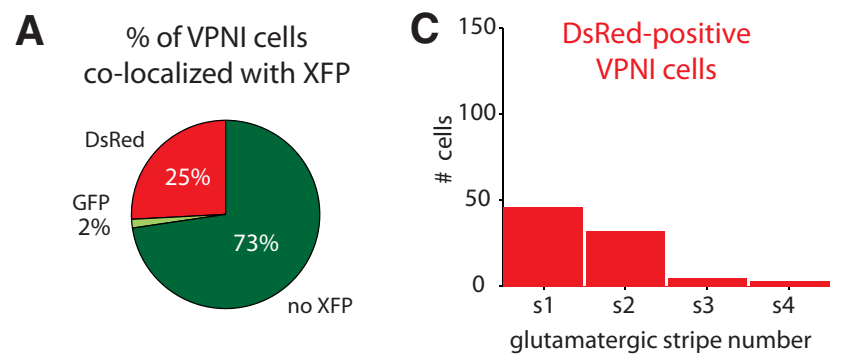

B Spatial distribution
of DsRed-positive cells
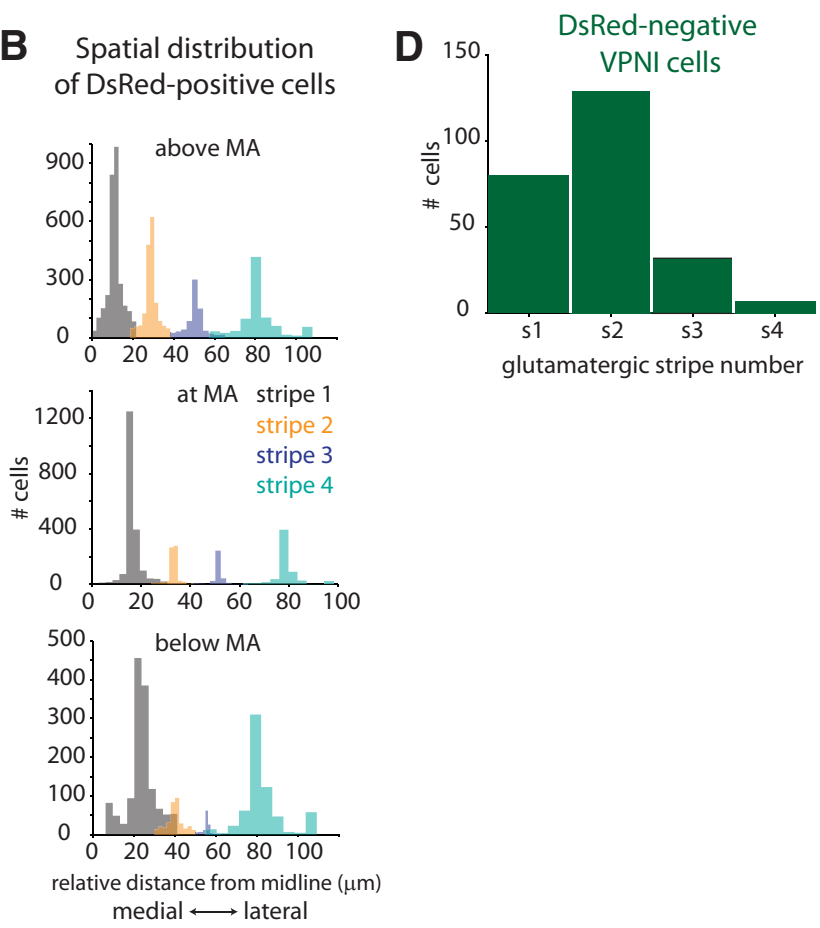

Figure 3. Colocalization of integrator cells with the genotypic scaffold. $\boldsymbol{A}$, Percentage of VPNI cells colocalized with vglut2:DsRed (red), glyt2:GFP (light green), or not colocalized with either fluorescent protein (dark green). $\boldsymbol{B}, \mathrm{ML}$ distribution of DsRed-positive cells along glutamatergic stripes relative to midline (see Materials and Methods). Some distributions overlap (e.g., ventral stripes 1 and 2) because the stripe peaks converge at the caudal part of the hindbrain and distributions are averaged across multiple planes. C, D, VPNI cell localization to glutamatergic stripes 1-4 (denoted s1, s2, s3, s4). C, Number of DsRed-positive VPNI cells along glutamatergic stripes. $\boldsymbol{D}$, Number of DsRed-negative VPNI cells along the glutamatergic stripes.

cells from the dual-transgenic fish. In total, 86 DsRed-positive VPNI cells from 7 fish were included in the analysis. First, we quantified the stripe-like nature of the hindbrain patterning qualitatively observed in Figure $1 C-E$ by assigning all DsRedpositive cells (regardless of VPNI function) to different stripe regions based on proximity to the stripe peaks identified at each plane (like those in Fig. 2A; see Materials and Methods). DsRedpositive cells were indeed clustered into four regions along the ML axis (Fig. 3B), with relative percentages along stripes 1 to 4 of $48.0 \%, 28.7 \%, 9.9 \%$, and $13.5 \%$, respectively (note that due to limited sampling of the lateral edges of the hindbrain in $\mathrm{rh} 7$, the cell counts along the fourth, most lateral, glutamatergic stripe may have been underestimated). Next, we found that, among the DsRed-positive VPNI cell group, most neurons were distributed along the first glutamatergic stripe (53.5\%), with progressively fewer cells along the second (37.2\%), third (5.8\%), and fourth
(3.5\%) stripes (Fig. 3C). These results indicate the presence of a large population of excitatory VPNI cells along the first glutamatergic stripe, comprised of cells that are colocalized with the alx transcription factor; and a smaller population of excitatory VPNI cells along the second glutamatergic stripe coinciding with the $d b \times 1 b$ transcription factor domain.

We also examined the distribution of DsRed-negative VPNI cells relative to the glutamatergic stripes. From this set, we expected that the DsRed-negative VPNI population ( $n=247$ cells) could be composed of two groups: glutamatergic cells that were not DsRed expressing because of mosaicism and neurons that were neither glutamatergic nor glycinergic. We found that most DsRed-negative cells were distributed along the second glutamatergic stripe $(51.8 \%)$, with progressively fewer cells along stripes 1 $(32.4 \%), 3(13.0 \%)$, and $4(2.8 \%)$ (Fig. $3 D)$. The difference in the distributions of DsRed-positive and -negative cells $(p=5.6 \times$ $10^{-3}$, Kolmogorov-Smirnov [KS] test; $p=8.87 \times 10^{-4}$, Wilcoxon rank sum test) suggests that the DsRed-negative group includes one or more nonglutamatergic populations likely centered along stripe 2 .

To further examine this possibility, we assessed, for the first and second stripes, differences between the spatial distributions of the DsRed-positive and -negative VPNI cell groups along the DV and RC dimensions (Fig. 4). The spatial distributions along stripe 1 generally appeared similar, both along the DV (Fig. 4A; $p=0.16, \mathrm{KS}$ test) and RC dimensions (Fig. $4 B ; p=0.08, \mathrm{KS}$ test). By contrast, the spatial distributions along stripe 2 were notably different, with a relative prevalence of DsRed-negative cells at ventral (Fig. $4 C ; p=1.32 \times 10^{-9}$, KS test) and caudal (Fig. $4 D$; $\mathrm{RC}: p=0.017$, KS test) positions. Because no significant differences were observed in the spatial distributions of DsRed-positive and -negative cells along stripe 1, we henceforth treat the VPNI cells along stripe 1 as one population. On the other hand, these findings support the idea of a nonglutamatergic VPNI population located primarily along or in proximity to glutamatergic stripe 2 .

Next, we examined the identity and composition of the nonglutamatergic VPNI population. First, given that these cells are not glutamatergic and unlikely to be glycinergic, we asked what their neurotransmitter type could be. Previous work in the rat nucleus prepositus hypoglossi (NPH), a hindbrain nucleus with neurons homologous to teleost VPNI cells (Aksay et al., 2000), found that most neurons are either glutamatergic or GABAergic, and few cells $(<5 \%)$ are of cholinergic or glycinergic type (Shino et al., 2008). Furthermore, in situ staining in 4-5 dpf zebrafish showed that transcripts for glycine transporter 2 ( glyt2) and glutamate decarboxylase $(G A D)$ are colocalized in the hindbrain of larval zebrafish (Higashijima et al., 2004), with dual-stained cells becoming either glycinergic or GABAergic as the fish develops. To determine whether GABAergic cells are present in the caudal hindbrain where nonglutamatergic, nonglycinergic VPNI cells were found, we performed in $5 \mathrm{dpf} v g l u t 2$ :GFP transgenic animals whole-mount immunostaining for GABA and GFP. In our stainings (Fig. 5A-C), the cellular GABA and GFP signals were mutually exclusive, suggesting that the a-GABA stain and vglut2:GFP line were specific for GABAergic and glutamatergic cells, respectively. In rhombomeres 7 and 8 (Fig. $5 A^{\prime}-C^{\prime}$ ), we found that a large GABAergic population was evident just lateral of the first glutamatergic stripe and intermingled with the second glutamatergic stripe, consistent with a GABAergic VPNI population located within the $d b \times 1 b$ transcription factor domain.

Based on this finding and previous work in the rabbit NPH suggesting that GABAergic NPH cells send contralateral projections (Arts et al., 2000), we conjectured that a significant portion 


\section{Stripe-specific spatial distribution of VPNI cells}
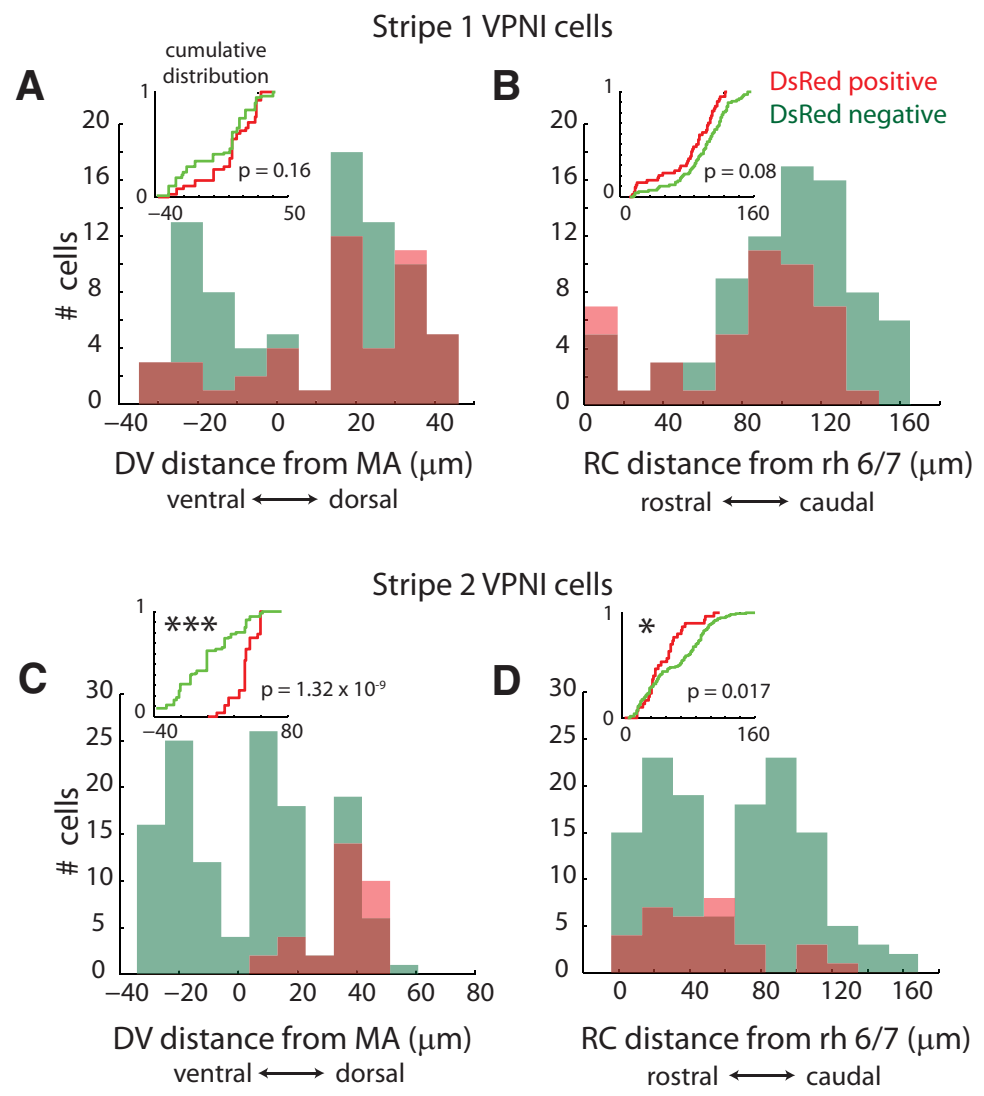

Figure 4. Spatial distribution of VPNI cells along glutamatergic stripes 1 and 2. DsRed-positive and -negative cell counts are indicated in red and dark green, respectively. $\boldsymbol{A}, \boldsymbol{C}$, DV location relative to level of MAs of VPNI cells along stripes 1 and 2, respectively. $\boldsymbol{B}, \boldsymbol{D}, \mathrm{RC}$ distance from the rh $6 / 7$ border of VPNI cells along stripes 1 and 2 , respectively. Insets, Cumulative distributions. ${ }^{*} 10^{-2}<p<0.05$. $^{* * *} p<10^{-4}$.

of the DsRed-negative population along stripe 2 consisted of contralaterally projecting GABAergic neurons. We showed further support for this idea through unilateral injections into the caudal hindbrain of the calcium sensitive dye rhodamine 2-AM ( rhod 2; Fig. 5D-F), an indicator we previously observed tended to give quite spatially localized loading (data not shown), and which we therefore reasoned would highlight via axonal loading a putative contralaterally projecting GABAergic population along stripe 2 on the contralateral side. Unilateral rhod 2 injections (Fig. $5 F$, white star) resulted, on the ipsilateral side, in labeling of VPNI neurons on all four stripes, but on the contralateral side, only in labeling VPNI neurons along stripe 2 ( $n=19$ fish). The colocalization of contralaterally loaded VPNI neurons and a sizeable GABAergic population suggests the presence of a GABAergic $d b x 1 b$ VPNI subtype.

Together, these results support the presence of three major subpopulations of cells in the VPNI: one medially located glutamatergic group associated with the alx transcription factor, a second more laterally located glutamatergic group within the dorsal $d b \times 1 b$ transcription factor domain, and a third putative GABAergic group distributed throughout the $d b \times 1 b$ domain.

\section{Morphological features}

We next set out to identify the key anatomical features of the three VPNI subpopulations, focusing on axonal projection and termination patterns, and dendritic elaborations $(n=29$ cells).
To do so for glutamatergic VPNI neurons, we sampled the morphology of cells from the first and second glutamatergic stripes in $\mathrm{Tg}$ (vglut2:DsRed) fish via targeted single-cell electroporation with Alexa-488 dextran. We further examined the morphology of functionally identified alx VPNI neurons in separate $\mathrm{Tg}$ (vglut2: DsRed) fish loaded with OGB. To examine the morphology of putative GABAergic neurons, we targeted in separate fish loaded with rhod 2 functionally identified ventrally positioned VPNI neurons located contralateral of the injection site.

Glutamatergic cells associated with the alx domain in rhombomeres $7-8(n=8)$ often had ascending projections consistent with those expected for excitatory VPNI neurons $(n=5)$. Figure 6 shows the main morphological features of two such filled neurons. The first cell (Fig. 6A-D) had aspiny dendrites directed laterally and medially, with the medially directed ones crossing the midline (Fig. 6A,B). The axon initiated directly from the soma (Fig. $6 B)$, bifurcated to a short descending portion that collateralized and terminated in the caudal hindbrain (Fig. $6 B$, inset) and a longer ascending branch that also had local collaterals within rh 7-8 (Fig. 6B). The ascending branch then coursed ventrally toward and past the abducens motor nucleus, giving rise to collaterals near the abducens nucleus (Fig. 6B, C) and ventral of the cerebellum (Fig. $6 D$ ), before terminating near the midbrain-hindbrain border in the valvula below the optic tectum (data not shown). The second cell (Fig. $6 E-H$ ) also had aspiny dendrites directed laterally and medially (no midline crossings), with a long lateral-caudal dendritic branch (Fig. $6 F$, inset). The axon split off from the initial segment of the main dendrite, sent a collateral with en passant boutons to rh 7-8 (Fig. 6F, inset), and then coursed ventrally and rostrally, sending collaterals at the abducens nucleus (Fig. 6G) and terminating ventral of the cerebellum (Fig. $6 H$ ).

We next reconstructed the morphology of these 5 neurons (see Materials and Methods); Figure 7 shows projections onto the horizontal plane from four of these cells, with axons and somata indicated in red and dendrites a shade lighter. The reconstructions underestimated the morphological complexity of the cells, as the reconstruction algorithm sometimes missed thin axon collaterals and small dendritic branchlets. Most cells had extensive dendritic arbors, with some crossing of the midline ( $n=3$; cells 1 , 2 , and 4 ). All 5 of the cells had both ascending and descending axonal segments which each gave rise to collaterals in rh 7-8. The ascending branch often terminated in rh 4, 5, or 6 near the vestibular or abducens nuclei ( $n=3$; e.g., cells 1 and 4 ) or extended rostral of rh 4 , terminating in or past the cerebellum $(n=2$; cells 2 and 3 ). The descending branches of these cells either terminated in the hindbrain $(n=3$; e.g., cells 2 and 3$)$ or spinal cord ( $n=1$, cell 4 ; the termination site for cell 1 could not be determined). The compendium at the top of Figure 7 shows the localization of these cells' somata relative to the abducens 


\section{GABAergic neuron distribution along stripe 2}
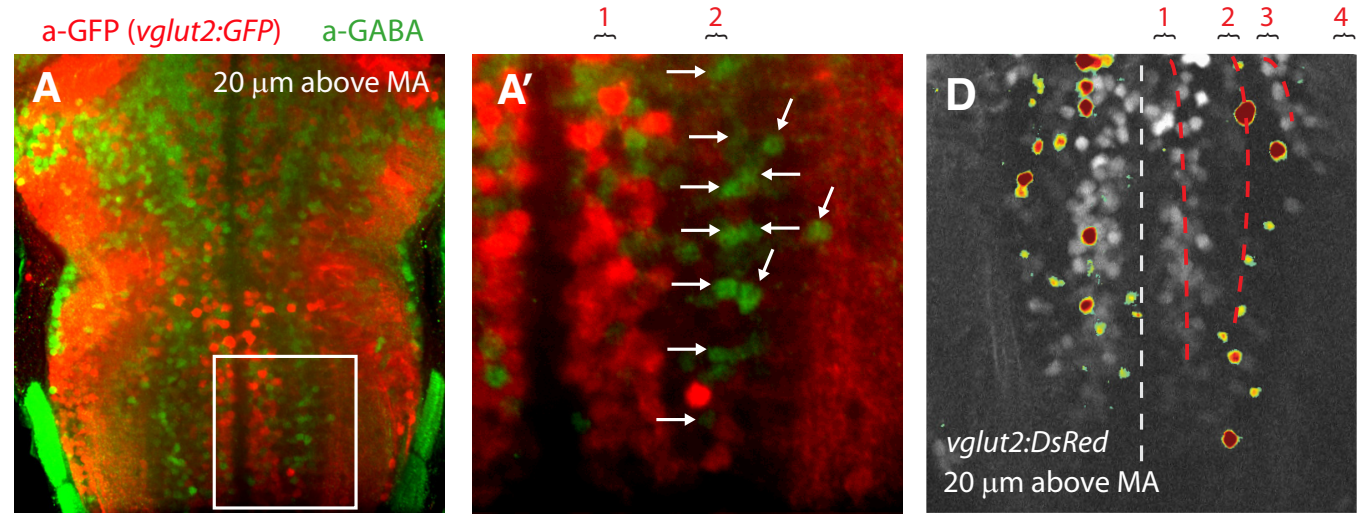

$1 \quad 2$
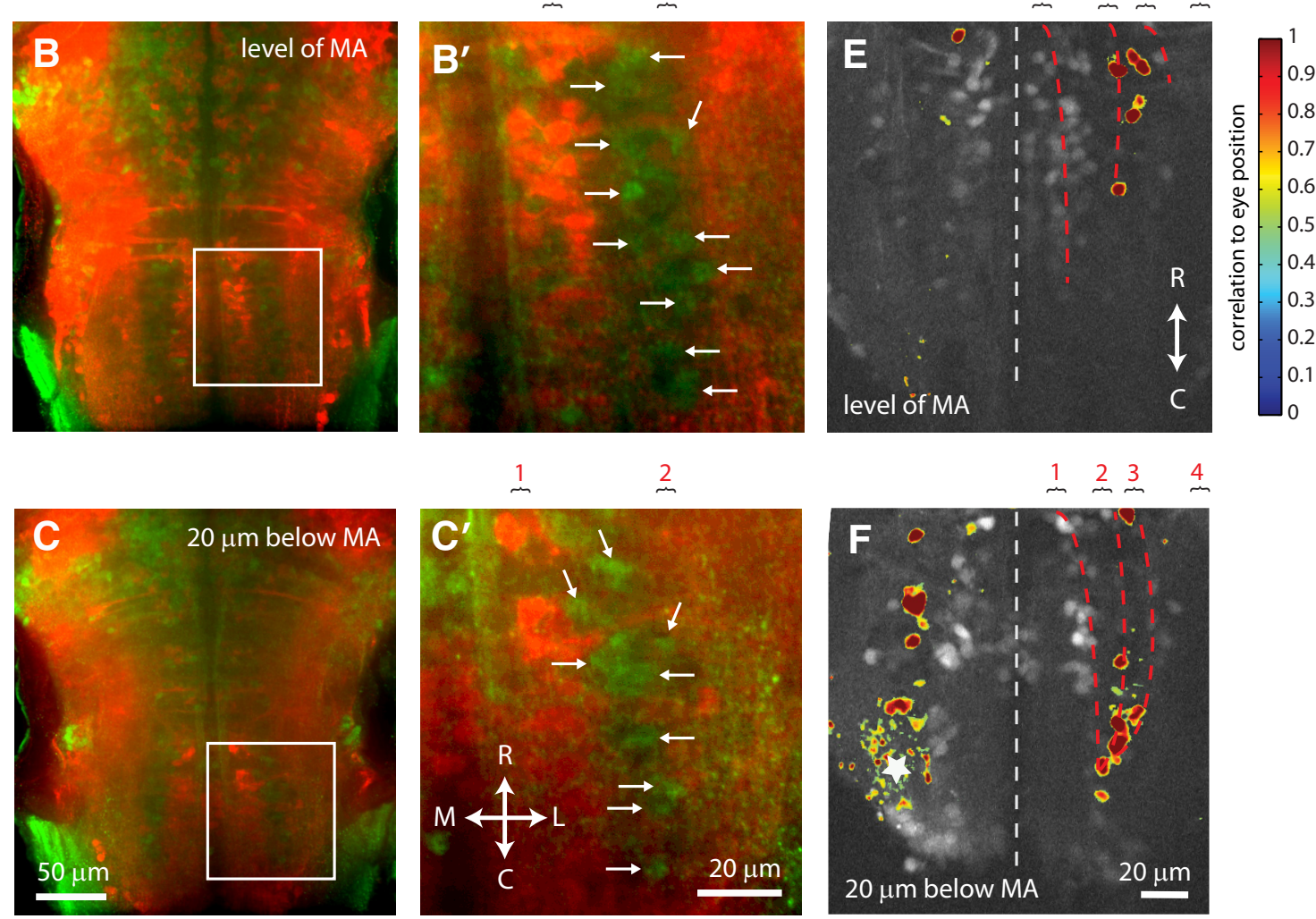

Figure 5. Location of a putative GABAergic VPNI subpopulation. $A-C$, Dorsal view of a 5 dpf vglut2:GFP larva stained for a-GABA (green) and a-GFP (red) at three different DV depths. $A^{\prime}-C^{\prime}$, Insets, Highlight of GABA-positive cells lateral of the first glutamatergic stripe and intermingled with the second glutamatergic stripe (white arrows). $\boldsymbol{D}-\boldsymbol{F}$, Localized loading of contralaterally projecting VPNI cells with calcium indicator rhod 2-AM. Heat maps showing pixels correlated with eye position or velocity during optokinetic response, superimposed on vglut2:DsRed expression pattern in rh 7-8. All colored pixels have correlation coefficient of at least 0.5 . Glutamatergic stripes are indicated at the top of each panel, and stripe peaks are marked with dashed red lines. $\boldsymbol{F}$, White star represents approximate site of injection.

motoneurons in rhombomeres 5 and 6 as identified in separate experiments using the transgenic mnxl:Tag-RFP- $t$ line (Jao et al., 2012).

Next, we verified that the general features observed above for glutamatergic alx neurons with ascending axonal branches accurately reflected the morphological properties of alx VPNI neurons. In a smaller set of experiments, functionally identified neurons in $\mathrm{Tg}$ (vglut2:DsRed) fish bulk-loaded with OGB were targeted for single-cell electroporation $(n=4)$. These cell soma were located near the caudal end of the alx stripe and 5-10 $\mu \mathrm{m}$ dorsal of the level of the MA. Figure 8 shows the activity and morphological features of two of these neurons. The fluorescence activity of these cells (blue) was highly correlated to eye position (black) during constant velocity (Fig. 8A,E) and sinusoidally varying (Fig. $8 B, F$ ) optokinetic stimulus (stimulus position, gray), with a high degree of saccade sensitivity. All four cells had extensive dendritic arbors, many of which stratified in a multipolar manner, with dendrites largely directed ventromedially and ventrolaterally, and some with medial branches crossing the midline $(n=2$; e.g., the cell in Fig. $8 C, D)$. All four cells also had extensive and complex axonal projections, all of which emanated from the primary dendritic branch, had multiple bifurcations and prominent ascending branches that gave rise to axon collaterals or terminals in rh 5-6 near the abducens nucleus (Fig. 


\section{Morphological features of Stripe 1 glutamatergic neurons}
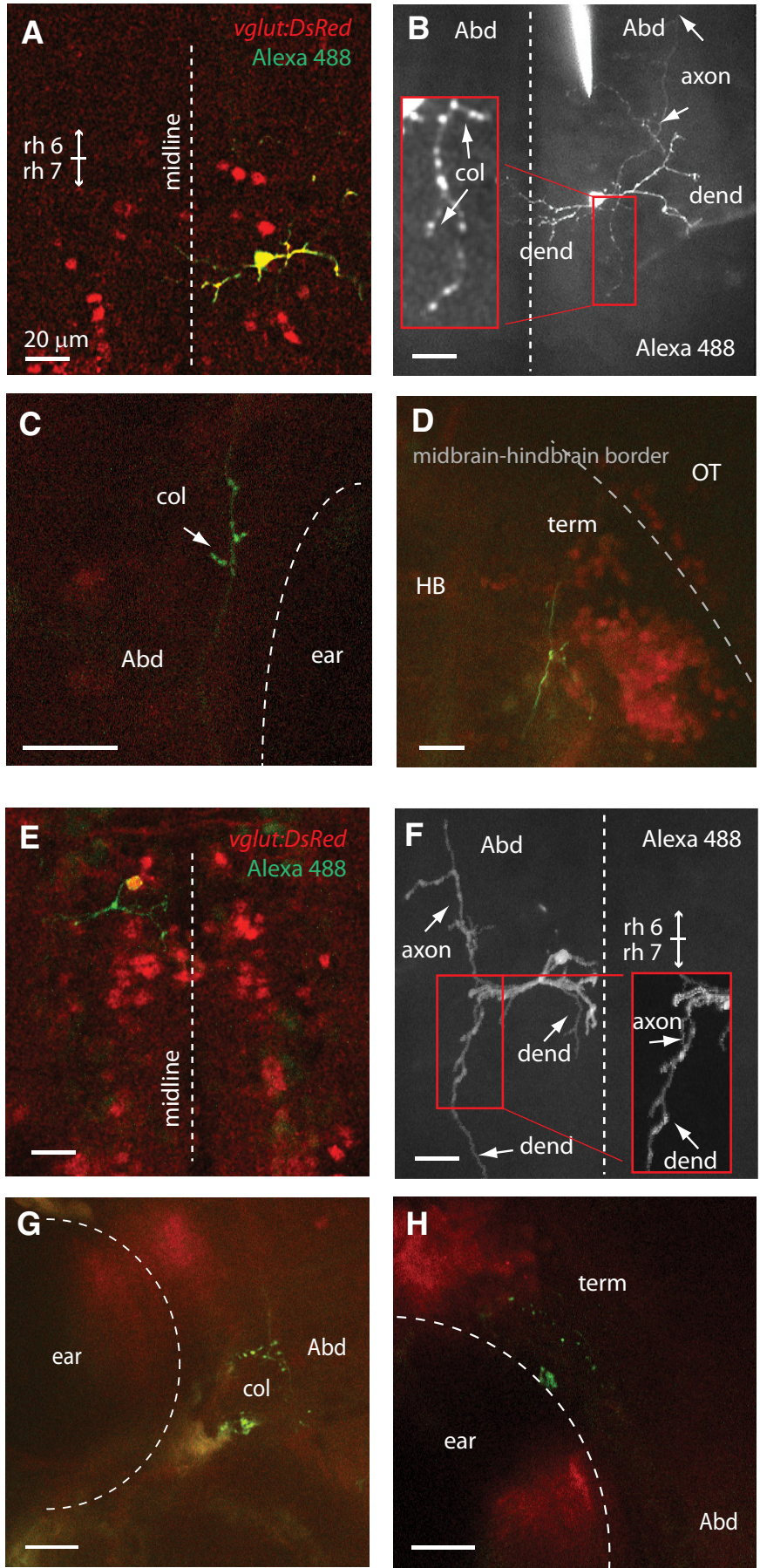

Figure 6. Anatomy of two glutamatergic alx cells. $\boldsymbol{A}, \boldsymbol{E}$, Location of filled cells (yellow-green) on the medial glutamatergic stripe (red). $\boldsymbol{B}, \boldsymbol{F}$, Dendrites (dend) of both cells ramify in the medial and lateral directions, with dendrites of the first cell crossing the midline. Axons have both ascending and descending segments, and the predominantly ascending portions give rise to collaterals (col) near or at the abducens motor nucleus (Abd). $\boldsymbol{C}, \boldsymbol{D}, \mathbf{G}, \boldsymbol{H}$, Higher-magnification images of axonal segments and collaterals near the abducens nucleus in the hindbrain $(\mathrm{HB})$ and terminating (term) near the midbrain-hindbrain border near the optic tectum (OT). In all images, rostral is to the top, caudal toward the bottom. Scale bars, $20 \mu \mathrm{m}$.

$\left.8 C^{\prime}, G^{\prime}\right)$. In addition, local collaterals and en passant boutons could be found in three of the cells in locations ventral of the soma (e.g., Fig. $8 D, H$, insets); the axon of the fourth cell was faintly labeled, making it difficult to identify thin collaterals or small boutons. Together, these findings identify as key morphological features of the glutamatergic alx VPNI subpopulation ex- tensive dendritic arbors and ascending axonal projections with collaterals and en passant boutons that overlap with the dendritic fields of VPNI cells and that terminate in expected targets of the VPNI.

We next targeted DsRed-positive cells along the second glutamatergic $d b \times 1 b$ stripe $(n=5)$. The reconstructions of two representative cells are shown in Figure 9 (cells 1 and 2, orange). All of the cells had laterally directed, aspiny dendritic arbors. Contrary to expectations for excitatory VPNI cells (Aksay et al., 2000, 2007), all of the cells labeled had contralateral projections. The axons initiated directly from the somata and opposite from the dendrites, coursed medially to cross the midline, then projected rostrally and ventrally. In 4 of 5 cases, collaterals were only observed in regions rostral of the caudal hindbrain rh 7-8; in 1 case, collaterals were seen both rostral and caudal of the border between rh 6 and 7. Among 3 cells for which full axonal projections could be traced, all showed collaterals or terminations at the level of the abducens motor nucleus. The key morphological features of the glutamatergic $d b \times 1 b$ VPNI class thus consist of relatively compact, laterally directed dendritic arbors and contralateral axonal projections, giving rise to collaterals predominantly rostral of the dendritic fields of VPNI cells, near the abducens nuclei.

Next, we targeted functionally identified VPNI cells that were loaded from contralateral injections of the calcium indicator rhod 2 and were ventrally located along glutamatergic stripe $2(n=12)$. Representative cells from this putative GABAergic $d b \times 1 b$ subpopulation are shown in Figure 9 (cells 3 and 4, green). The cells exhibited relatively stereotyped morphological features: dendrites were generally aspiny and directed laterally, axons initiated from the medial side of the somata, coursed medially to cross the midline, and then gave rise to collaterals with en passant boutons within rh 7-8 on the contralateral side before ascending with terminations at or past rh 5-6. Of 5 cells that were carefully inspected for projections rostral of the abducens motor nuclei, all appeared to terminate in rh 5-6. The schematic at the top of Figure 9 shows the approximate locations of these representative cells from groups within the $d b \times 1 b$ domain: their DV positions (not indicated in the figure) are consistent with the spatial distribution shown in Figure $4 C$, with somata of targeted glutamatergic cells located dorsal of the MA and those of GABAergic cells ventral of the MA. In summary, although the glutamatergic and GABAergic $d b \times 1 b$ subpopulations share common morphological features such as laterally directed dendritic elaborations and contralateral 
axonal projections, they differ in that the axons of the putative GABAergic cells give rise to collaterals in the contralateral dendritic fields of VPNI cells, whereas those of the glutamatergic cells primarily send collaterals at targets rostral of the contralateral VPNI dendritic field.

Our examination of the morphological features of the three major VPNI subpopulations has begun to create concrete links between the molecular-genetic indicators of cell identity and systems-level functional roles hinging on patterns of connectivity. First, we found that the morphological features of the glutamatergic neurons associated with the alx domain support a principal role in the generation of persistent firing through local axo-dendritic synaptic interactions (Kamath and Keller, 1976; Seung et al., 2000; Aksay et al., 2003, 2007). These neurons also appear to provide the primary source of excitatory drive for tonic changes in abducens motoneuron firing and yoked changes in eye position. Second, examining the morphological features of the glutamatergic neurons associated with the $d b \times 1 b$ domain led to the discovery of a new class of excitatory VPNI cells with contralateral ascending projections and little or no collateralization in rh 7-8, suggesting that these are readout neurons potentially involved in the generation of disconjugate movements, such as those engaged during vergence (Mays, 1984; Zhang et al., 1991; Cullen and Van Horn, 2011), monocular control (Debowy and Baker, 2011), or in the control of eye plant dynamics through cocontraction (Robinson, 1964; Hendel et al., 2008). Last, our findings related to putative GABAergic $d b x 1 b$ cells suggest that these neurons provide the hypothesized pathway for mediating coupling between the bilateral VPNI populations (Aksay et al., 2003, 2007).

\section{Morphological features of Stripe 1 glutamatergic neurons}

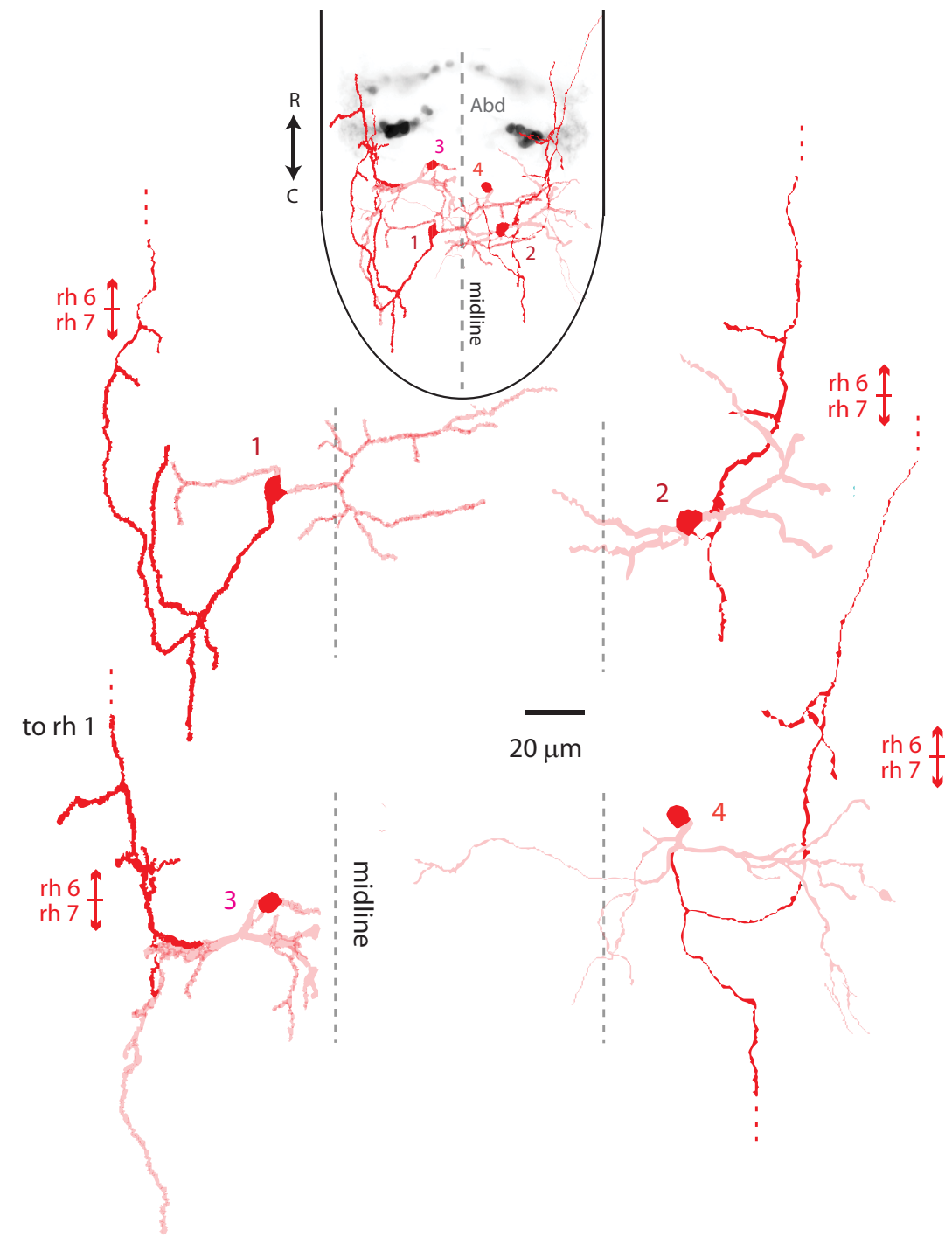

Figure 7. Reconstructions of glutamatergic cells in the alx domain. Red neurites represent axons. Double-sided arrows indicate the rh $6 / 7$ border $\sim 30-40 \mu \mathrm{m}$ below the MA level where most axon branchings and collaterals were observed. Light red represents dendrites. Dotted lines indicate the midline. Schematic at top, Approximate RC ordering and cell locations relative to the abducens motor nuclei (Abd) in rhombomeres 5 and 6 . Abd labeling is from the $\operatorname{Tg}(m n x 1: \operatorname{Tag}-R F P-t)$ line. Although the $R C$ ordering of the cells is correct, the cells' exact RC positions were not registered across fish. Dots indicate where the full extent of axonal projections are not shown. Morphological features of cell 2 are shown in panels Figure $6 \boldsymbol{A}-\boldsymbol{D}$, and those of cell 3 are shown in Figure $6 E-H$.

\section{Patterns of persistent firing dynamics across the scaffold}

We next aimed to examine whether there were links between the structural-genotypic scaffold and the patterns of persistent firing of integrator neurons. Previous work found that, during fixation behavior, VPNI cells exhibit a range of persistence time constants (Miri et al., 2011b; Daie et al., 2015). Here we sought to determine whether variations in the dynamics of persistent activity during fixation behavior were specific to particular VPNI subgroups. To do so, we first determined the estimated firing rate response of each cell by deconvolving its smoothed saccade-triggeredaverage fluorescence (see Materials and Methods) (Fig. 10A,B). We then measured the dynamics of persistent firing by: first fitting the firing rate response during the first $5 \mathrm{~s}$ of fixation with an exponential of time constant $\tau$, and second by calculating the area under normalized exponential fits to these firing rates to determine a persistent firing metric $\rho$; $\rho$ approached 0 for cells with weak persistence, was $\sim 0.5$ for those with intermediate persistence (corresponding to a $\tau$ value of $2.7 \mathrm{~s}$ ), was 1 for those that were perfectly persistent, and was $>1$ for cells that were unstable (corresponding to negative $\tau$ values). We present results using the $\rho$ measure as it was less sensitive to noise than $\tau$, the traditional metric for persistent activity, especially for very persistent cells.

The distribution of dynamics across the entire VPNI population confirmed previous observations. Across the entire scaffold, the mean and median $\rho$ for the VPNI were 0.66 and 0.64 , respectively. The least persistent $20 \%$ of cells had an average $\rho$ of 0.19 and the most persistent $20 \%$ of cells had an average $\rho$ of $1.18(n=344$ cells with qualifying fits; see Materials and Methods) (Fig. 10C), defining a persistence range for the population that was similar to previous observations (Miri et al., 2011b; Daie et al., 2015).

We next examined the persistence distribution of the VPNI subpopulations to determine whether the previously observed 


\section{Morphological features of functionally-identified Stripe 1 VPNI neurons}

A

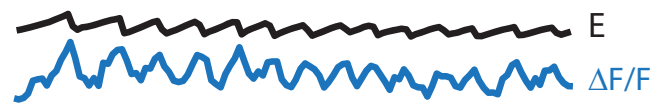

B

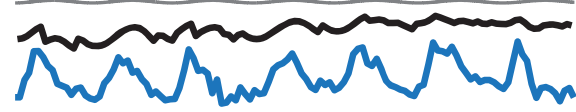

E

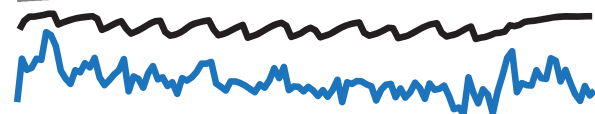

$\mathbf{F}$

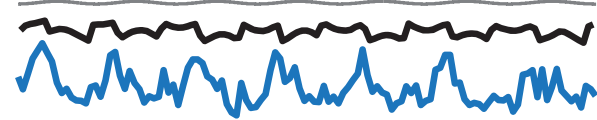

C

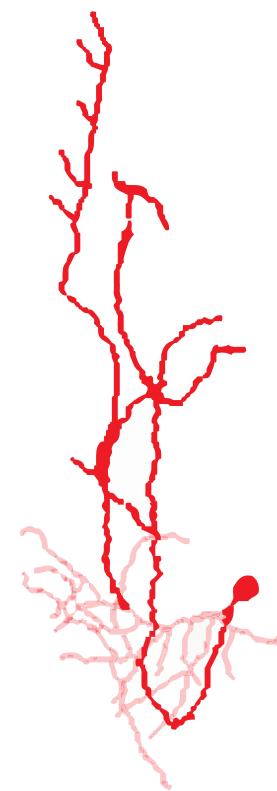

:

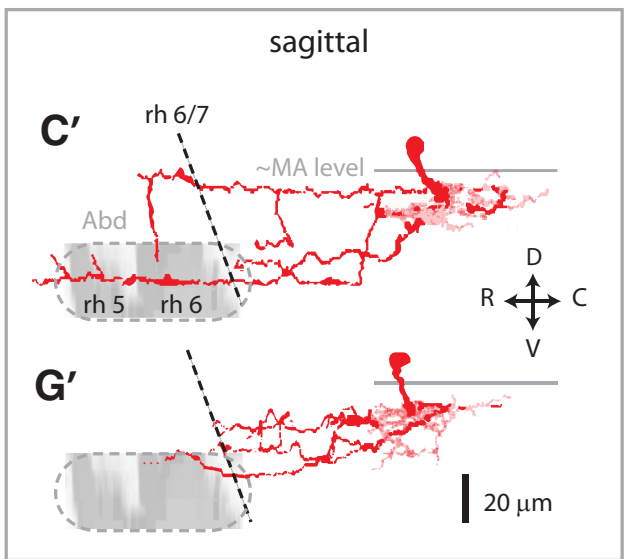

G

$$
\begin{array}{r}
50 \% \Delta \mathrm{F} / \mathrm{F}, \\
\frac{50^{\circ}}{20 \mathrm{sec}}
\end{array}
$$
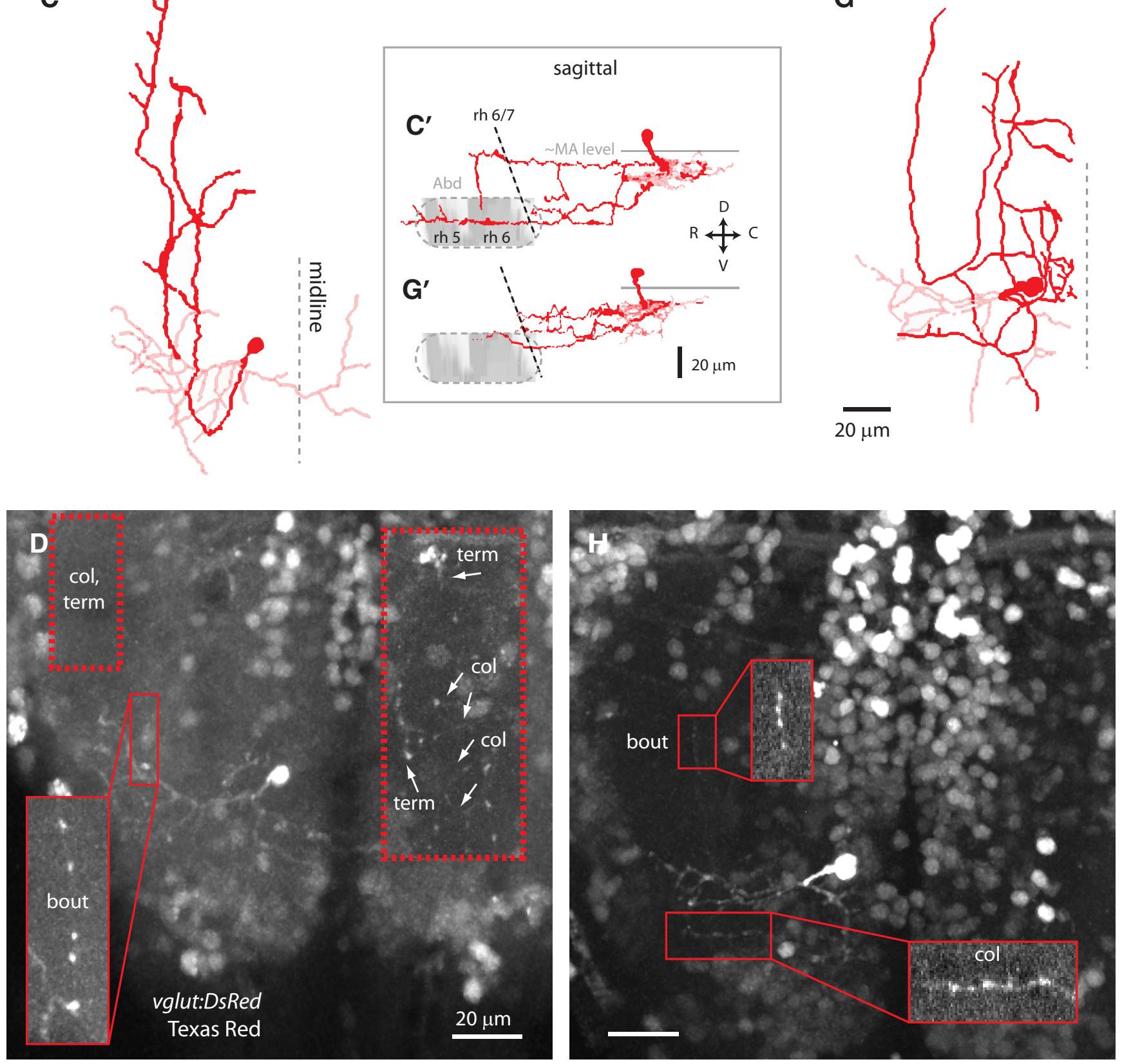
heterogeneity in persistence times arose from within or between subgroups. The heterogeneity in persistence observed globally was present in all three subpopulations. The mean and median $\rho$ among cells in the alx stripe were 0.72 and 0.74 , respectively $(n=134)$, and $\rho$ spanned a range of 0.21-1.29 (Fig. 10D). Among DsRed-positive $d b \times 1 b$ cells, $\rho$ had a mean and median of 0.42 and $0.27(n=26)$ and a range of $0.12-0.91$ (Fig. 10E, orange). DsRed-negative $d b \times 1 b$ cells had a mean and median $\rho$ of 0.68 and 0.65 , respectively $(n=116)$, and a persistence range of $0.24-1.13$ (Fig. 10E, green). We next compared the distributions of $\rho$ between each of the subpopulations. No significant differences were found between the alx and DsRed-negative $d b \times 1 b$ subgroups ( $p=$ $0.95, \mathrm{KS}$ test). However, $\rho$ values for the DsRed-positive $d b \times 1 b$ subgroup were significantly smaller than both the alx (Fig. $10 F$, red asterisks; $p=1.8 \times 10^{-3}$, KS test; $p=2.15 \times 10^{-4}$, rank sum) and DsRednegative $d b \times 1 b$ subgroups $(p=6.48 \times$ $10^{-4}$, green asterisks; KS test; $p=2.99 \times$ $10^{-4}$, rank sum). These results reveal that heterogeneity in persistence times is present among all three subpopulations, but there are significantly faster dynamics among cells in the DsRed-positive $d b \times 1 b$ subgroup.

Together, these results suggest that the functional organization of the VPNI may be linked, at least in part, to the structuralgenotypic scaffold. Our results suggest that recurrent excitatory interactions among glutamatergic neurons associated with the alx domain generate positive feedback that leads to heterogeneous persistent firing in this subpopulation. The distribution of dynamics generated in the alx domain appears to be read out by cells associated with the $d b \times 1 b$ domain; however, the glutamatergic cells in the $d b \times 1 b$ domain appear to have a bias toward shorter persistence times.

\section{Discussion}

We mapped the larval zebrafish oculomotor VPNI onto a genotypic scaffold, specifying the formative structure-function rela-

Figure 8. Morphological features of two functionally identified VPNI alx cells. A-D, Structure-function details of cell 1. $\boldsymbol{E}-\boldsymbol{H}$, Structure-function details of cell 2. Stimulus position (S, gray), eye position (E, black), and fluorescence responses $(\Delta \mathrm{F} / \mathrm{F}$, blue) of electroporated cells during $(\boldsymbol{A}, \boldsymbol{E})$ constant velocity and $(\boldsymbol{B}, \boldsymbol{F})$ sinusoidal optokinetic response. Reconstructions $(\boldsymbol{C}, \boldsymbol{G})$ and maximum-intensity projections $(\boldsymbol{D}, \boldsymbol{H})$ of filled cells. $\boldsymbol{C}^{\prime}, \boldsymbol{G}^{\prime}$, Insets, Sagittal views of the cells, with DV location relative to the MA level (gray line) and $R C$ location relative to the rh $6 / 7$ border (dashed black line). Gray represents the abducens motor nuclei (Abd). $\boldsymbol{D}, \boldsymbol{H}$, Insets, Time-averaged projections of single planes or maximum-intensity projections across a few planes (5-10 $\mu \mathrm{m}$ along DV dimension) spanning some of the cells' axon collaterals (col), terminals (term), or en passant boutons (bout). $\boldsymbol{D}$, Dashed rectangle on the left represents the approximate $x y$ location of the region shown in the dashed inset at right in which axon collaterals and terminals were found; this region was located ventrally and appears averaged out in the maximum projection.
Morphological features of Stripe 2 glutamatergic and putative GABAergic neurons

Figure 9. Reconstructions of cells along the $d b \times 1 b$ stripe. Representative glutamatergic cells 1 and 2 have axons (orange) that laterally, give collaterals within rh 7-8 in the dendritic field of contralateral VPNI neurons, and then project rostrally to (axons in green). Double-sided arrows indicate the $\mathrm{rh} 6 / 7$ border at the DV level of the nuclei (Abd). Although the RC ordering of the cells is correct, the cells' exact RC positions were not registered across fish. Dots

tionships underlying temporal integration in the oculomotor system. We provided evidence for three major VPNI subpopulations: a medially located excitatory glutamatergic alx group, a more lateral excitatory glutamatergic $d b \times 1 b$ group, and a lateral, putative GABAergic inhibitory $d b x 1 b$ group. Targeted electroporation provided further structural and connectivity information, suggesting specific roles for each subpopulation. These findings help to establish concrete links between circuit-level dynamics and the physiological and anatomical properties of the VPNI's constitutive neurons.

VPNI neurons in the alx transcription factor domain appear to provide excitatory drive to the ipsilateral abducens motoneurons and may play an important role in the generation of persistent firing. Many VPNI neurons were glutamatergic and found at medial positions consistent with localization in the alx transcription factor domain. Glutamatergic neurons in this most medial stripe had ipsilaterally projecting axons, in agreement with previous work on the morphology of hindbrain (Kinkhabwala et al., 2011) and spinal cord (Lewis, 2006) alx (chx10) neurons. Most cells had axons that ascended and collateralized in rhombomeres 5-6 near abducens motoneurons and interneurons, consistent with an oculomotor role. alx VPNI cells also had local axon collaterals in the VPNI dendritic field, suggesting that glutamatergic 


\section{Stripe-specific organization of VPNI neuron dynamics}

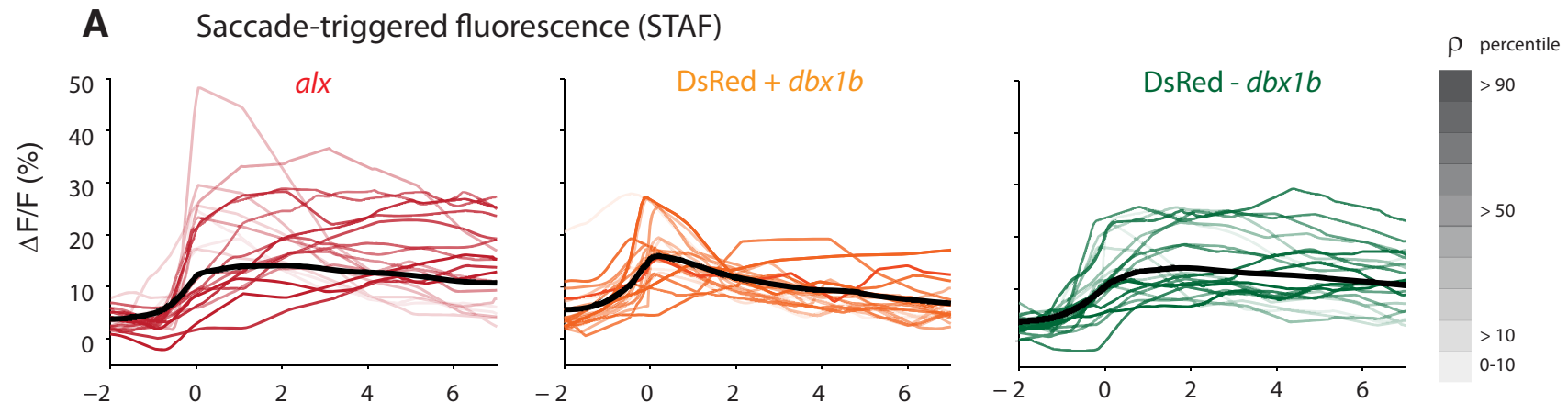

B Fitting deconvolved, smoothed fluorescence to estimate neuronal persistence, $\rho$
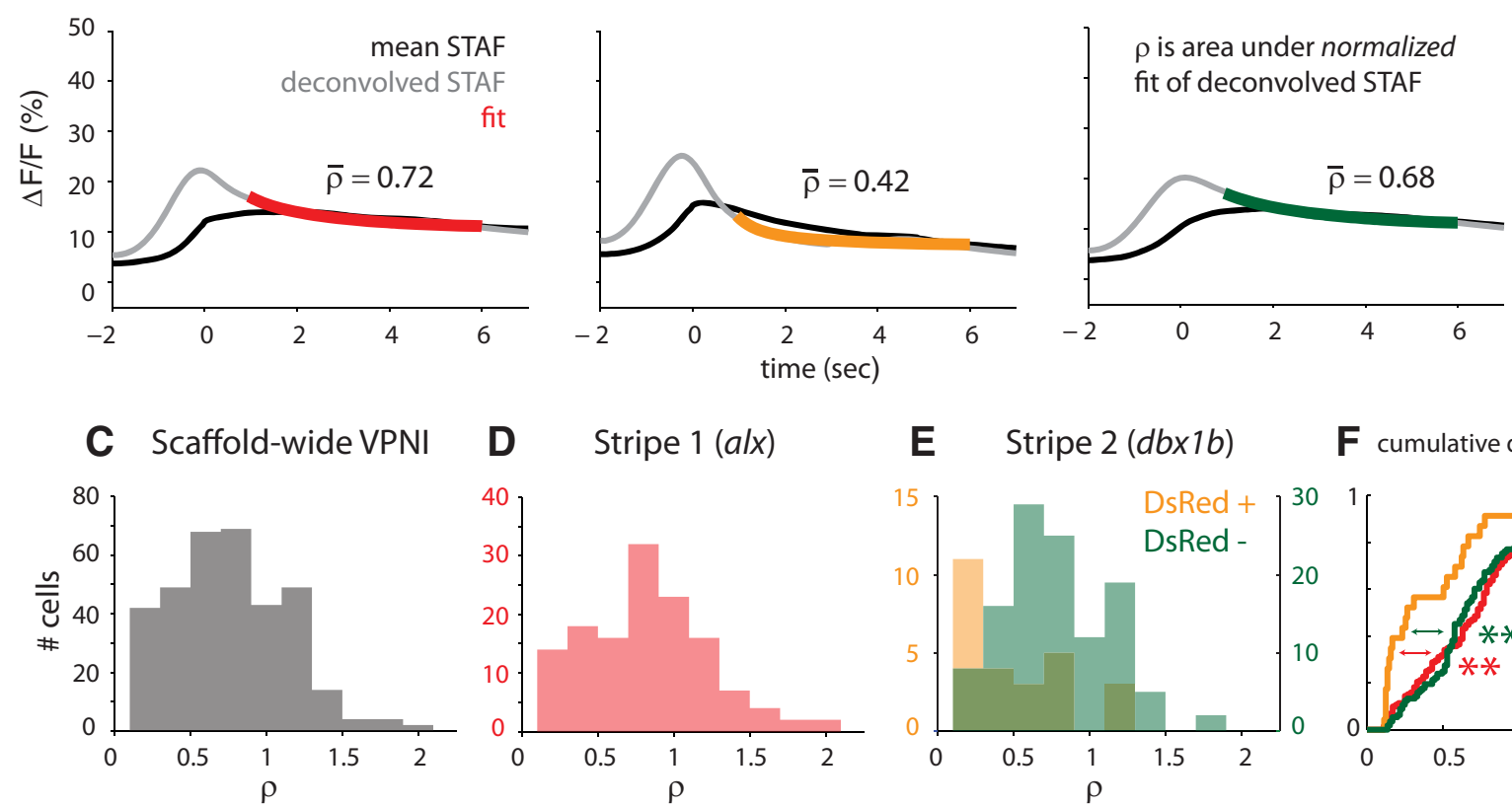

F cumulative distributions

Figure 10. Persistence heterogeneity across the VPNI and within VPNI subpopulations. $A$, STAF responses of a subset of cells in each subpopulation, chosen from a distribution sampling approximately uniformly across percentile rank of $\rho$ (indicated by the color intensity and summarized by the gray color scale at right; cells for which the percentile is $>90$ have persistence $\rho>90 \%$ of the cells). Black represents population-averaged STAFs. B, Population-averaged STAF (black), population-averaged deconvolved STAF (gray), and population-averaged fit (red, orange, green) to the deconvolved STAF. $\bar{\rho}$ is the population-averaged $\rho$. Distributions of persistence measure $\rho(\boldsymbol{C})$ across the VPNI, $(\boldsymbol{D})$ within the alx stripe, and $(\boldsymbol{E})$ between DsRed- positive and -negative subpopulations along the $d b \times 1 b$ domain. $\boldsymbol{F}$, Cumulative distributions of $\rho$ for the VPNI subpopulations. ${ }^{* *} 10^{-4}<p<10^{-2}$.

alx VPNI cells establish recurrent excitatory connections. Such connections are thought to play a critical role in generating positive feedback that supports persistent firing and temporal integration (Fisher et al., 2013; Joshua et al., 2013).

The above characteristics, when viewed in light of results from other species, begin to provide a detailed profile of a VPNI neuron class instrumental to the generation of persistent firing. With regard to the morphology of this class, in vivo studies of the goldfish VPNI identified a subpopulation with ipsilateral axonal projections to the abducens complex (Aksay et al., 2000). These neurons were associated with the ipsilaterally projecting "principal" neurons of the mammalian NPH (McCrea and Baker, 1985), a key component of the mammalian VPNI (Cannon and Robinson, 1987). These principal neurons were characterized by dendrites with uniform branching geometry and local axon collaterals with boutons en passant, consistent with our findings. With regard to network function, ipsilateral projections to the abducens motor nucleus have been hypothesized to be excitatory: in the goldfish, integrator and abducens motor neurons on the same side of the brain increase their firing rates together (with ipsilateral eye movements) (Aksay et al., 2000); and in the cat, excitatory field potentials at the abducens nucleus are triggered by firing in the ipsilateral NPH (Escudero et al., 1992). Our findings now indicate that this projection indeed is excitatory, and more specifically, is glutamatergic. With regard to intrinsic properties, in vitro electrophysiology of rat (Shino et al., 2008) and mouse (Kolkman et al., 2011) NPH revealed that glutamatergic neurons were most often associated with fast action potentials, a pronounced slow component of after-hyperpolarization (most often biphasic), and continuous, graded responses to current injection. These features characterize the "Type B" neuron identified in earlier in vitro recordings from guinea pig NPH (Idoux et al., 2006), and also match those of a small subpopulation of goldfish VPNI neurons recorded in vivo (Aksay et al., 2001). Together, these findings across multiple species suggest the following profile for a VPNI subpopulation critical for the generation of per- 
sistent firing: these cells are specified in part by the alx $(\operatorname{ch} x 10)$ transcription factor; they are glutamatergic, generally with fast action potentials and slow biphasic afterhyperpolarization; and they project ipsilaterally to the abducens nucleus, with local axon collaterals potentially supporting recurrent excitation.

Putative GABAergic VPNI neurons in the $d b x 1 b$ transcription factor domain appear to provide inhibitory drive to the contralateral abducens nucleus and are likely responsible for coordinating persistent firing between bilateral VPNI populations. We observed very few glycinergic VPNI neurons in rhombomeres 7-8. Instead, many VPNI neurons were in a ventral GABAergic cell zone localized within the more lateral $d b x 1 b$ transcription factor domain. Putative GABAergic VPNI neurons had contralaterally projecting axons consistent with hindbrain (Kinkhabwala et al., 2011) and spinal cord (Lewis, 2006; Satou et al., 2012) $d b x 1 b$ neurons. They further presented collaterals in the primary dendritic field of contralateral VPNI neurons and subsequently projected rostrally to the abducens nucleus. These features suggest that GABAergic VPNI neurons play a role in coordination of two half-integrators (Aksay et al., 2007; Fisher et al., 2013).

Together with results from other species, the above findings help to delineate a second important VPNI cell class. With regards to the morphology of this second class, previous work in the goldfish has shown that some VPNI neurons project across midline and present collaterals in the contralateral VPNI and abducens nucleus, aligning them with the contralaterally projecting principal neurons of the NPH (Aksay et al., 2000). Functionally, contralateral VPNI projections are hypothesized to be inhibitory: in the goldfish, the firing of a left VPNI neuron increases as the firing of right VPNI and abducens motor neurons decrease; and in the cat, inhibitory field potentials at the abducens nucleus are triggered by firing in the contralateral NPH (Escudero et al., 1992). Our findings indicate this cell class indeed is inhibitory and, in the zebrafish, GABAergic. This agrees with data from rabbit (Highstein, 1973), rat (Nomura et al., 1984), and guinea pig (Kumoi et al., 1987), suggesting primarily GABAergic inhibitory inputs to the abducens nucleus, and findings that commissural NPH neurons in the rabbit are predominantly GABAergic (Arts et al., 2000). Although glycinergic somatic inputs to the abducens nucleus have previously been highlighted in several species (Spencer et al., 1989; Straka and Dieringer, 1993; Graf et al., 1997), these studies have also found GABAergic distal targets in the neuropil, consistent with our finding of lateral VPNI axonal fields in the neuropil of the abducens motor neurons. With regard to intrinsic properties, in vitro recordings of rat (Shino et al., 2008) and mouse (Kolkman et al., 2011) NPH revealed two major GABAergic NPH subpopulations: those displaying "Type B" characteristics, and those exhibiting slower action potentials and briefer after-hyperpolarization (always monophasic) in a phenotype termed "Type A" (Idoux et al., 2006). Interestingly, the characteristic after-hyperpolarization of Type A cells was also observed in the majority of in vivo recordings from goldfish VPNI neurons (Aksay et al., 2001). Hence, given that glutamatergic VPNI neurons appear to be predominantly of the Type B category and that Type B neurons seem to be a minority in the VPNI (Aksay et al., 2001), it would appear by exclusion that most GABAergic VPNI neurons would be associated with the Type A classification. Together, these findings suggest a second major VPNI subpopulation: these neurons are GABAergic, located within the $d b \times 1 b$ transcription factor domain, most often show slow action potentials and brief monophasic afterhyperpolariza-
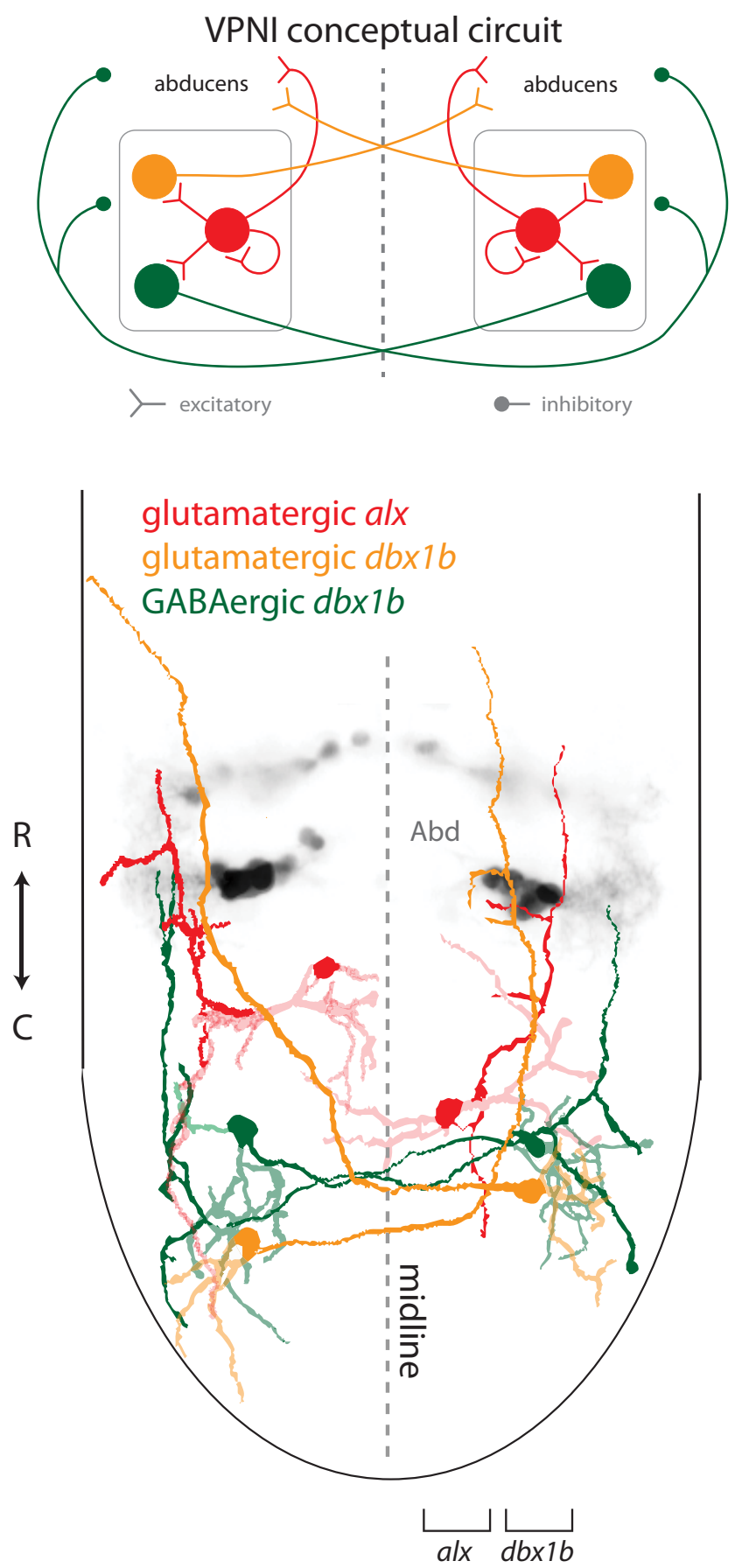

Figure 11. Summary linking connectivity, neurotransmitter, and transcription factor identity of three identified VPNI cell types. Top, Conceptual circuit diagram of the VPNI supporting an augmented hypothesis for the mechanism of integration involving ipsilaterally projecting glutatmatergic alx (red), contralaterally projecting glutamatergic $d b \times 1 b$ (orange), and commissural putative GABAergic $d b \times 1 b$ (dark green) neurons. Open and filled terminations represent excitatory and inhibitory connections, respectively. Bottom, Maximum projections of reconstructions of representative cells from the three VPNI classes. Although the RC ordering of the cells is correct, the cells' exact RC positions were not registered across fish. The full extent of axonal projections are not shown.

tion, and have contralateral projections to opposing VPNI neurons and the abducens motoneurons.

A third, smaller group of VPNI neurons appears primarily to play a role in readout from the integrator. These neurons were glutamatergic, located within the $d b \times 1 b$ domain, and had axons 
that projected to the contralateral abducens nucleus. Interestingly, this cell group seldom gave rise to collaterals within rhombomeres 7-8, suggesting the primary role for this VPNI cell type is to provide readout to motoneuron pools. Because output from these neurons to the abducens motor nuclei does not align with the push-pull organization established by the above glutamatergic al $x$ and GABAergic $d b x 1 b$ cell groups, their exact role is open to speculation. These cells could play a role in vergence and/or disconjugate eye movements, perhaps by supplementing the activity of near-response and/or saccadic-burst neurons to allow the eyes to rotate in opposite directions and redirect gaze between near and far targets (Mays, 1984; Cullen and Van Horn, 2011). Alternatively, these cells could be involved in monocular control (Debowy and Baker, 2011), or in controlling the dynamics of the eye plant by enabling cocontraction of the extraocular muscles (Sklavos et al., 2005).

Although the data presented here clearly support the presence of three VPNI subpopulations, further work may be necessary to better delineate the relative abundances of these subpopulations and to explore the possibility of additional VPNI subgroups. Although the dye loading achieved through bolus injection of OGB appeared to be uniform over the caudal hindbrain, it is possible that anisotropies in loading may have led to some sampling bias. Furthermore, given that our dataset undersampled from the lateral-most fourth glutamatergic stripe, there may exist a VPNI subpopulation from this group, possibly associated with the barhl2 transcription factor domain (Kinkhabwala et al., 2011). Finally, given that GABAergic cells appear to be sparsely distributed within the al $x$ stripe (Fig. $5 A-C$ ), the possibility remains that some of the DsRed-negative alx cells comprise a distinct colocalized GABAergic VPNI subgroup, possibly arising from a progenitor common to that of the alx glutamatergic pool (Kimura et al., 2008). Moreover, given the ipsilateral axonal profiles of cells in the alx domain, it is possible that such a cell class may play a role in stabilization of persistent firing via negative feedback (Boerlin et al., 2013; Lim and Goldman, 2013). Future work in transgenic animals with ubiquitously expressed calcium sensors and XFP labeling of GABAergic neurons (Satou et al., 2013) might help to further elucidate links between the hindbrain scaffold and VPNI function.

Overall, the results presented help to clarify three aspects of how persistent activity is generated and coordinated in the VPNI. First, previous studies suggested that persistent firing in the VPNI is generated primarily by positive feedback through recurrent excitatory connections. This is supported by recordings showing changes in persistent firing correlate with sustained changes in membrane-potential fluctuations (Aksay et al., 2001), positive correlations in the firing of neighboring VPNI neurons (Aksay et al., 2003; Miri et al., 2011b; Dale and Cullen, 2015), and deficits in high-rate persistent firing following silencing of neighboring VPNI cells (Aksay et al., 2007). The present study suggests that neurons of the glutamatergic alx subclass have the requisite functional and structural properties needed to mediate recurrent excitation and positive feedback in support of persistent firing and temporal integration (Fig. 11, red). Second, previous work suggested that inhibitory commissural connections subserve coordination between two half-integrators that independently generate persistent activity. This hypothesis is supported by negative cross-correlations between bilateral cell pairs (Aksay et al., 2003), deficits in low-rate persistent firing following inactivation of contralateral VPNI neurons (Aksay et al., 2007), and computational modeling (Fisher et al., 2013). The present work suggests that GABAergic $d b \times 1 b$ neurons mediate these inhibitory interactions between the two halves of the VPNI (Fig. 11, green). Finally, our results suggest that glutamatergic $d b \times 1 b$ VPNI neurons form a third subpopulation, one with a role in readout from the integrator (Fig. 11, orange). Together, these results suggest the form of a structural and genotypic scaffold underlying temporal integration in the VPNI, and provide information crucial to the design of future experiments testing hypothesized mechanisms of integration using targeted perturbations (Arrenberg et al., 2009; Gonçalves et al., 2014).

\section{References}

Aksay E, Baker R, Seung HS, Tank DW (2000) Anatomy and discharge properties of pre-motor neurons in the goldfish medulla that have eyeposition signals during fixations. J Neurophysiol 84:1035-1049. Medline

Aksay E, Gamkrelidze G, Seung HS, Baker R, Tank DW (2001) In vivo intracellular recording and perturbation of persistent activity in a neural integrator. Nat Neurosci 4:184-193. CrossRef Medline

Aksay E, Baker R, Seung HS, Tank DW (2003) Correlated discharge among cell pairs within the oculomotor horizontal velocity-to-position integrator. J Neurosci 23:10852-10858. Medline

Aksay E, Olasagasti I, Mensh BD, Baker R, Goldman MS, Tank DW (2007) Functional dissection of circuitry in a neural integrator. Nat Neurosci 10:494-504. CrossRef Medline

Arrenberg AB, Del Bene F, Baier H (2009) Optical control of zebrafish behavior with halorhodopsin. Proc Natl Acad Sci U S A 106:17968-17973. CrossRef Medline

Arts MP, De Zeeuw CI, Lips J, Rosbak E, Simpson JI (2000) Effects of nucleus prepositus hypoglossi lesions on visual climbing fiber activity in the rabbit flocculus. J Neurophysiol 84:2552-2563. Medline

Bae YK, Kani S, Shimizu T, Tanabe K, Nojima H, Kimura Y, Higashijima S, Hibi M (2009) Anatomy of zebrafish cerebellum and screen for mutations affecting its development. Dev Biol 330:406-426. CrossRef Medline

Bagwell CB, Adams EG (1993) Fluorescence spectral overlap compensation for any number of flow cytometry parameters. Ann N Y Acad Sci 677: 167-184. CrossRef Medline

Baird GS, Zacharias DA, Tsien RY (2000) Biochemistry, mutagenesis, and oligomerization of DsRed, a red fluorescent protein from coral. Proc Natl Acad Sci U S A 97:11984-11989. CrossRef Medline

Beck JC, Rothnie P, Straka H, Wearne SL, Baker R (2006) Precerebellar hindbrain neurons encoding eye velocity during vestibular and optokinetic behavior in the goldfish. J Neurophysiol 96:1370-1382. CrossRef Medline

Boerlin M, Machens CK, Denève S (2013) Predictive coding of dynamical variables in balanced spiking networks. PLoS Comput Biol 9:e1003258. CrossRef Medline

Brondi M, Sato SS, Rossi LF, Ferrara S, Ratto GM (2012) Finding a needle in a haystack: identification of EGFP tagged neurons during calcium imaging by means of two-photon spectral separation. Front Mol Neurosci 5:96. CrossRef Medline

Cannon SC, Robinson DA (1987) Loss of the neural integrator of the oculomotor system from brain stem lesions in monkey. J Neurophysiol 57: 1383-1409. Medline

Cullen KE, Van Horn MR (2011) The neural control of fast vs slow vergence eye movements. Eur J Neurosci 33:2147-2154. CrossRef Medline

Daie K, Goldman MS, Aksay ER (2015) Spatial patterns of persistent neural activity vary with the behavioral context of short-term memory. Neuron 85:847-860. CrossRef Medline

Dale A, Cullen KE (2015) Local population synchrony and the encoding of eye position in the primate neural integrator. J Neurosci 35:4287-4295. CrossRef Medline

Debowy O, Baker R (2011) Encoding of eye position in the goldfish horizontal oculomotor neural integrator. J Neurophysiol 105:896-909. CrossRef Medline

Drobizhev M, Tillo S, Makarov NS, Hughes TE, Rebane A (2009) Absolute two-photon absorption spectra and two-photon brightness of orange and red fluorescent proteins. J Phys Chem B 113:855-859. CrossRef Medline

Escudero M, de la Cruz RR, Delgado-García JM (1992) A physiological study of vestibular and prepositus hypoglossi neurones projecting to the abducens nucleus in the alert cat. J Physiol 458:539-560. CrossRef Medline

Fisher D, Olasagasti I, Tank DW, Aksay ER, Goldman MS (2013) A modeling 
framework for deriving the structural and functional architecture of a shortterm memory microcircuit. Neuron 79:987-1000. CrossRef Medline

Fuchs AF, Kaneko CR, Scudder CA (1985) Brainstem control of saccadic eye movements. Annu Rev Neurosci 8:307-337. CrossRef Medline

Gonçalves PJ, Arrenberg AB, Hablitzel B, Baier H, Machens CK (2014) Optogenetic perturbations reveal the dynamics of an oculomotor integrator. Front Neural Circuits 8:10. CrossRef Medline

GrafW, Spencer R, Baker H, Baker R (1997) Excitatory and inhibitory pathways to the extraocular motor nuclei in goldfish. J Neurophysiol 77:27652779. Medline

Guizar-Sicairos M, Thurman ST, Fienup JR (2008) Efficient subpixel image registration algorithms. Opt Lett 33:156-158. CrossRef Medline

Heim R, Cubitt AB, Tsien RY (1995) Improved green fluorescence. Nature 373:663-664. CrossRef Medline

Hendel T, Mank M, Schnell B, Griesbeck O, Borst A, Reiff DF (2008) Fluorescence changes of genetic calcium indicators and OGB-1 correlated with neural activity and calcium in vivo and in vitro. J Neurosci 28:73997411. CrossRef Medline

Higashijima S, Mandel G, Fetcho JR (2004) Distribution of prospective glutamatergic, glycinergic, and GABAergic neurons in embryonic and larval zebrafish. J Comp Neurol 480:1-18. CrossRef Medline

Highstein SM (1973) Synaptic linkage in the vestibulo-ocular and cerebellovestibular pathways to the VIth nucleus in the rabbit. Exp Brain Res 17:301-314. Medline

Idoux E, Serafin M, Fort P, Vidal PP, Beraneck M, Vibert N, Mühlethaler M, Moore LE (2006) Oscillatory and intrinsic membrane properties of guinea pig nucleus prepositus hypoglossi neurons in vitro. J Neurophysiol 96:175-196. CrossRef Medline

Jao LE, Appel B, Wente SR (2012) A zebrafish model of lethal congenital contracture syndrome 1 reveals Gle1 function in spinal neural precursor survival and motor axon arborization. Development 139:1316-1326. CrossRef Medline

Joshua M, Medina JF, Lisberger SG (2013) Diversity of neural responses in the brainstem during smooth pursuit eye movements constrains the circuit mechanisms of neural integration. J Neurosci 33:6633-6647. CrossRef Medline

Kamath BY, Keller EL (1976) A neurological integrator for the oculomotor control system. Math Biosci 30:341-352. CrossRef

Kimura Y, Satou C, Higashijima S (2008) V2a and V2b neurons are generated by the final divisions of pair-producing progenitors in the zebrafish spinal cord. Development 135:3001-3005. CrossRef Medline

Kinkhabwala A, Riley M, Koyama M, Monen J, Satou C, Kimura Y, Higashijima S, Fetcho J (2011) A structural and functional ground plan for neurons in the hindbrain of zebrafish. Proc Natl Acad Sci US A 108:1164-1169. CrossRef Medline

Kolkman KE, Moghadam SH, du Lac S (2011) Intrinsic physiology of identified neurons in the prepositus hypoglossi and medial vestibular nuclei. J Vestib Res 21:33-47. CrossRef Medline

Koyama M, Kinkhabwala A, Satou C, Higashijima S, Fetcho J (2011) Mapping a sensory-motor network onto a structural and functional ground plan in the hindbrain. Proc Natl Acad Sci U S A 108:1170-1175. CrossRef Medline

Kumoi K, Saito N, Tanaka C (1987) Immunohistochemical localization of gamma-aminobutyric acid- and aspartate-containing neurons in the guinea pig vestibular nuclei. Brain Res 416:22-33. CrossRef Medline

Lewis KE (2006) How do genes regulate simple behaviours? Understanding how different neurons in the vertebrate spinal cord are genetically specified. Philos Trans R Soc Lond B Biol Sci 361:45-66. CrossRef Medline

Lim S, Goldman MS (2013) Balanced cortical microcircuitry for maintaining information in working memory. Nat Neurosci 16:1306-1314. CrossRef Medline

Longair MH, Baker DA, Armstrong JD (2011) Simple Neurite Tracer: open source software for reconstruction, visualization and analysis of neuronal processes. Bioinformatics 27:2453-2454. CrossRef Medline

Mays LE (1984) Neural control of vergence eye movements: convergence and divergence neurons in midbrain. J Neurophysiol 51:1091-1108. Medline

McCrea RA, Baker R (1985) Anatomical connections of the nucleus prepositus of the cat. J Comp Neurol 237.

McLean DL, Fan J, Higashijima S, Hale ME, Fetcho JR (2007) A topographic map of recruitment in spinal cord. Nature 446:71-75. CrossRef Medline

Miri A, Daie K, Burdine RD, Aksay E, Tank DW (2011a) Regression-based identification of behavior-encoding neurons during large scale optical imaging of neural activity at cellular resolution. J Neurophysiol 105:964980. CrossRef Medline

Miri A, Daie K, Arrenberg AB, Baier H, Aksay E, Tank DW (2011b) Spatial gradients and multidimensional dynamics in a neural integrator circuit. Nat Neurosci 14:1150-1159. CrossRef Medline

Mütze J, Iyer V, Macklin JJ, Colonell J, Karsh B, Petrášek Z, Schwille P, Looger LL, Lavis LD, Harris TD (2012) Excitation spectra and brightness optimization of two-photon excited probes. Biophys J 102:934-944. CrossRef Medline

Nomura I, Senba E, Kubo T, Shiraishi T, Matsunaga T, Tohyama M, Shiotani Y, Wu JY (1984) Neuropeptides and gamma-aminobutyric acid in the vestibular nuclei of the rat: an immunohistochemical analysis. I. Distribution. Brain Res 311:109-118. CrossRef Medline

Raphan T, Cohen B (1978) Brainstem mechanisms for rapid and slow eye movements. Annu Rev Physiol 40:527-552. CrossRef Medline

Robinson DA (1964) The mechanics of human saccadic eye movement. J Physiol 174:245-264. CrossRef Medline

Robinson DA (1968) Eye movement control in primates. Science 161:12191224. CrossRef Medline

Robinson DA (1989) Integrating with neurons. Annu Rev Neurosci 12:3345. CrossRef Medline

Romo R, Brody CD, Hernández A, Lemus L (1999) Neuronal correlates of parametric working memory in the prefrontal cortex. Nature 399:470 473. CrossRef Medline

Satou C, Kimura Y, Higashijima S (2012) Generation of multiple classes of V0 neurons in zebrafish spinal cord: progenitor heterogeneity and temporal control of neuronal diversity. J Neurosci 32:1771-1783. CrossRef Medline

Satou C, Kimura Y, Hirata H, Suster ML, Kawakami K, Higashijima S (2013) Transgenic tools to characterize neuronal properties of discrete populations of zebrafish neurons. Development 140:3927-3931. CrossRef Medline

Scudder CA, Kaneko CS, Fuchs AF (2002) The brainstem burst generator for saccadic eye movements: a modern synthesis. Exp Brain Res 142:439_ 462. CrossRef Medline

Seung HS, Lee DD, Reis BY, Tank DW (2000) Stability of the memory of eye position in a recurrent network of conductance-based model neurons. Neuron 26:259-271. CrossRef Medline

Shadlen MN, Newsome WT (2001) Neural basis of a perceptual decision in the parietal cortex (area LIP) of the rhesus monkey. J Neurophysiol 86: 1916-1936. Medline

Shino M, Ozawa S, Furuya N, Saito Y (2008) Membrane properties of excitatory and inhibitory neurons in the rat prepositus hypoglossi nucleus. Eur J Neurosci 27:2413-2424. CrossRef Medline

Sklavos S, Porrill J, Kaneko CR, Dean P (2005) Evidence for wide range of time scales in oculomotor plant dynamics: implications for models of eye-movement control. Vision Res 45:1525-1542. CrossRef Medline

Spencer RF, Wenthold RJ, Baker R (1989) Evidence for glycine as an inhibitory neurotransmitter of vestibular, reticular, and prepositus hypoglossi neurons that project to the cat abducens nucleus. J Neurosci 9:2718 2736. Medline

Straka H, Dieringer N (1993) Electrophysiological and pharmacological characterization of vestibular inputs to identified frog abducens motoneurons and internuclear neurons in vitro. Eur J Neurosci 5:251-260. CrossRef Medline

Zhang Y, Gamlin PD, Mays LE (1991) Antidromic identification of midbrain near response cells projecting to the oculomotor nucleus. Exp Brain Res 84:525-528. CrossRef Medline 